\title{
Assessment of Immunological Features in Muscle-Invasive Bladder Cancer Prognosis Using Ensemble Learning
}

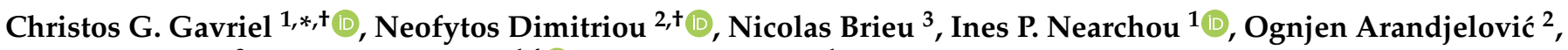 \\ Günter Schmidt ${ }^{3}$, David J. Harrison ${ }^{1,4}{ }^{(1)}$ and Peter D. Caie ${ }^{1}$ \\ 1 School of Medicine, University of St Andrews, St Andrews KY16 9TF, UK; i.p.nearchou@gmail.com (I.P.N.); \\ djh20@st-andrews.ac.uk (D.J.H.); pdc5@st-andrews.ac.uk (P.D.C.) \\ 2 School of Computer Science, University of St Andrews, St Andrews KY16 9SX, UK; \\ nd26@st-andrews.ac.uk (N.D.); oa7@st-andrews.ac.uk (O.A.) \\ 3 Definiens GmbH, 80636 Munich, Germany; nicolas.brieu@gmail.com (N.B.); gschmidt@definiens.com (G.S.) \\ 4 NHS Lothian, University Hospitals Division, Edinburgh EH16 4SA, UK \\ * Correspondence: christoscgav@gmail.com \\ + These authors contributed equally to this work.
}

check for

updates

Citation: Gavriel, C.G.; Dimitriou,

N.; Brieu, N.; Nearchou, I.P.;

Arandjelović, O.; Schmidt, G.;

Harrison, D.J.; Caie, P.D. Assessment

of Immunological Features in

Muscle-Invasive Bladder Cancer

Prognosis Using Ensemble Learning.

Cancers 2021, 13, 1624. https://

doi.org/10.3390/cancers13071624

Academic Editor: Bernard Malavaud

Received: 7 January 2021

Accepted: 16 March 2021

Published: 1 April 2021

Publisher's Note: MDPI stays neutral with regard to jurisdictional claims in published maps and institutional affiliations.

Copyright: (C) 2021 by the authors. Licensee MDPI, Basel, Switzerland. This article is an open access article distributed under the terms and conditions of the Creative Commons Attribution (CC BY) license (https:/ / creativecommons.org/licenses/by/ $4.0 /)$.
Simple Summary: Muscle-invasive bladder cancer (MIBC) accounts for the majority of bladder cancer mortality worldwide. Clinical assessment of MIBC mainly relies on the TNM staging system to provide guidance for both prognosis and therapy planning. Based on standardized quantification of tumour-immune features across whole slide images, and in conjunction with clinical information, we construct an ensemble machine learning model that correctly classifies $71.4 \%$ of the patients who succumb to MIBC, significantly higher than the $28.6 \%$ of TNM staging system. Post-hoc analysis of our model reveals clinically relevant, immunological features for MIBC prognosis, thereby further supporting their adoption into the clinic.

Abstract: The clinical staging and prognosis of muscle-invasive bladder cancer (MIBC) routinely includes the assessment of patient tissue samples by a pathologist. Recent studies corroborate the importance of image analysis in identifying and quantifying immunological markers from tissue samples that can provide further insight into patient prognosis. In this paper, we apply multiplex immunofluorescence to MIBC tissue sections to capture whole-slide images and quantify potential prognostic markers related to lymphocytes, macrophages, tumour buds, and PD-L1. We propose a machine-learning-based approach for the prediction of 5 year prognosis with different combinations of image, clinical, and spatial features. An ensemble model comprising several functionally different models successfully stratifies MIBC patients into two risk groups with high statistical significance $\left(p\right.$ value $<1 \times 10^{-5}$ ). Critical to improving MIBC survival rates, our method correctly classifies $71.4 \%$ of the patients who succumb to MIBC, which is significantly more than the $28.6 \%$ of the current clinical gold standard, the TNM staging system.

Keywords: immuno-oncology; tumour microenvironment; tumour budding; PD-L1; macrophages; lymphocytes; prognosis; survival analysis; machine learning; digital pathology

\section{Introduction}

Urothelial cancer of the bladder (bladder cancer) is one of the most prevalent cancers worldwide, with approximately 430,000 new diagnoses each year [1]. The high morbidity and mortality rates, as well as the high socioeconomic burden, make bladder cancer a debilitating and often fatal disease [2,3]. Even though the majority of bladder cancer patients are diagnosed with non-muscle-invasive bladder cancer (NMIBC), recurrence and progression of the disease may lead to muscle-invasive bladder cancer (MIBC) [4]. Approximately $25 \%$ of newly diagnosed patients have MIBC, and unlike NMIBC, these tumours are biologically aggressive with limited therapeutic options. Although radical cystectomy 
with bilateral pelvic lymph node dissection is the current gold-standard treatment for MIBC, more than $50 \%$ of MIBC patients die from metastatic disease within 5 years [5]. To decrease the mortality rates, patients with a high risk of disease-specific death need to be identified more precisely, thereby allowing for better patient management and new treatments to be tested in the high-risk group.

Due to the intra- and inter-tumoural heterogeneity of $\mathrm{MIBC}$, which is evident from the phenotypic and molecular diversity of tumour cells, choosing the most effective treatment for each patient is very challenging [6,7]. Currently, clinical assessment of bladder uses the Tumour-Node-Metastasis (TNM) staging system [8], where T describes the depth of invasion into the bladder wall, and $\mathrm{N}$ and $\mathrm{M}$ the presence or lack of node and distant metastasis, respectively. MIBC ranges from tumours which invade the detrusor muscle (T2), to tumours which spread to nearby organs (T4) [1,9]. Although TNM plays a critical role in guiding treatment planning, it remains an anatomy-based classification tool with patients of the same tumour stage experiencing a high variability in disease outcome [10,11].

The tumour mass comprises a heterogeneous population of cancer cells, and, together with a diverse group of resident and infiltrating host cells, they make up the tumourimmune microenvironment [12,13]. With the emergence of immuno-oncology, many cancer researchers have investigated the importance of the intra-tumoural host immune response within what is often an immunosuppressive tumour microenvironment [14,15]. Analysing the location, density, functional state, and organisation of the immune cell populations within the tumour landscape, often termed as the immune contexture, has become a fundamental step in identifying immune system characteristics that may be beneficial to patients [16] In particular, an increasing number of studies has shown the critical role of the immune contexture (not yet included in the TNM guidelines) in patient survivability, suggesting that it could be a valuable determinant of patient prognosis $[15,17]$. Motivated by multiple papers [18-21], we have investigated the prognostic role of tumour-infiltrating lymphocytes (TILs), tumour-associated macrophages (TAMs), tumour buds (TBs), and programmed cell death-ligand 1 (PD-L1) in MIBC patients.

Lymphocytes and macrophages are generally found either infiltrating into or surrounding the tumour mass, both the core and the invasive front. TILs can be divided into subpopulations by virtue of their specialised functions, surface cluster of differentiation (CD) molecules and, in certain circumstances, morphological features. Cytotoxic T-cells are the main effector cells in the anti-tumour T-cell response, with a large volume of studies showing that their presence in the tumour-immune microenvironment is strongly associated with prolonged survival in various types of cancer [22,23]. TAMs have also been identified as decisive factors in the orchestration of the tumour-immune microenvironment [24]. They can exhibit polarised phenotypes, with classically activated M1 and alternatively activated M2 subpopulations possessing anti-tumoural and pro-tumoural abilities, respectively [25]. In particular, during metastasis, M2 macrophages are recruited at distinct pre-metastatic niches, where they can promote tumour cell dissemination and disrupt the function of TILs [24].

Tumour budding is generally considered to be the first step of cancer metastasis, defined as the dissociation of isolated single cancer cells or discrete clusters of up to four cancer cells, predominantly from the invasive front of the tumour [26]. In the last decade, tumour budding has been widely investigated as a marker of aggressive tumour behaviour, due to its association with adverse clinicopathological characteristics and the epithelialmesenchymal transition $[27,28]$. As a result, tumour budding has been added to TNM as a supplementary prognostic factor for colorectal cancer [29]. However, without reliable quantitative methods, tumour-budding quantification is challenging, due to poor interobserver consistency [30,31].

Immune checkpoints are cell-surface receptors expressed by immune cells that modulate immune responses [32]. Complex interactions between the immune system and cancer, including the manipulation of immune checkpoints such as programmed cell death 1 (PD-1), enable tumour cells to evade immune surveillance. Specifically, PD-L1, which is secreted by tumour cells, binds to PD-1 expressed on the surface of TILs and suppresses 
their function to ensure the growth and development of tumour cells [33]. Although our understanding of the intricate and dynamic relationship between tumour cells and host cells is increasing, further characterization of the precise impact of tumour cells on their surroundings is needed.

In recent years, machine learning (ML) methodologies have been widely utilized for the construction of predictive models based on biological features [34-36]. Nevertheless, the number of papers which have adopted ML methodologies for survival analysis is limited [34,36-38]. In this paper, we employ an ML methodology to investigate the prognostic role of TILs, TAMs, TBs, PD-L1, and other clinicopathological factors in MIBC. The contribution of this paper is fourfold. To the best of our knowledge, this is the first study reporting the labelling of entire MIBC tissue sections with multiple fluorescence immune markers as well as the quantification of immunological features across both the tumour core and the invasive front of whole-slide immunofluorescence images. In addition, using image, spatial, and clinical features, our ML methodology improves the accuracy of the 5 year prognosis for MIBC patients by a large margin when compared to the current gold standard, TNM. Lastly, our findings reinforce the importance of the immune contexture in cancer prognosis, thereby further supporting its adoption in the clinic.

\section{Materials and Methods}

\subsection{Patients and Tissue Samples}

Tissue specimens from patients with MIBC who underwent radical cystectomy at Royal Infirmary and Western General Hospital in Edinburgh between the years 2006 and 2013 were collated into a cohort. Patients were excluded from this study either due to incomplete clinical records, extensive tissue section artefacts, or data censoring. The final study cohort was comprised of 78 patients. Archived formalin-fixed paraffin-embedded (FFPE) tissue blocks presenting the deepest invasion of cancer were selected for each patient based on both macroscopic and microscopic examination of haematoxylin and eosin (H\&E)-stained slides by a pathologist and a research scientist. The corresponding unstained tissue sections were collected from the NHS Lothian NRS BioResource Research Tissue Bank, conforming to protocols approved under the ethical status granted by the East of Scotland Research Ethics Service (Ethical Approval Ref: 10/S1402/33) and with written informed consent from all the patients. All experiments were performed in accordance with the relevant guidelines and regulations. Clinicopathological data that included age, sex and TNM stage status, along with survival data, were retrieved from the available electronic medical records. Patients were followed-up for a total time of 113 months, with a median survival time of 23.6 months. In order to maintain the anonymity of the patient information, the samples were de-identified prior to conducting this study.

\subsection{Multiplex Immunofluorescence and Whole Slide Imaging}

For each patient, automated tyramide-based immunofluorescence was performed on two de-paraffinised serial $3 \mu \mathrm{m}$ thick sections of FFPE tissue mounted on superfrost plus slides using a Dako link 48 autostainer (Dako, Agilent Technologies). Primary antibodies against Pan-cytokeratin (PanCK), CD3, CD8, CD68, CD163 and PD-L1 were used to label urothelial cells, general T-cells, cytotoxic T-cells, M1/M2 (total) macrophages, M2 macrophages and immune checkpoint ligand PD-L1, respectively (see Supplementary Table S1 for further details about the primary antibodies). To increase the detection sensitivity and to visualise the target protein, tyramide signal amplification (TSA) reagents FITC, CY3 and CY5 were used for CD3 or CD68, PD-L1, and CD8 or CD163 respectively. Alexa Fluor 750 conjugated streptavidin antibody was used for the detection of PanCK. See Supplementary Table S2 for the detection and visualization reagents. Nuclei were counterstained with Hoechst (Hoechst 33342, Cat\# H3570, ThermoFisher Scientific) and ProLong Gold Antifade mountant (Cat\# P36930, ThermoFisher Scientific) applied directly to the tissue samples. The multiplex immuno-labeled tissue slides were scanned at $20 \times$ magnification and digitized into whole-slide fluorescence images using a Carl Zeiss AxioScan.Z1 
scanner (Zeiss, Göttingen, Germany). Examples are shown in Figure 1. Supplementary Table S3 and Figure S1 show the excitation and emission wavelengths, along with the exposure time for each antibody used for multispectral imaging.

(a)

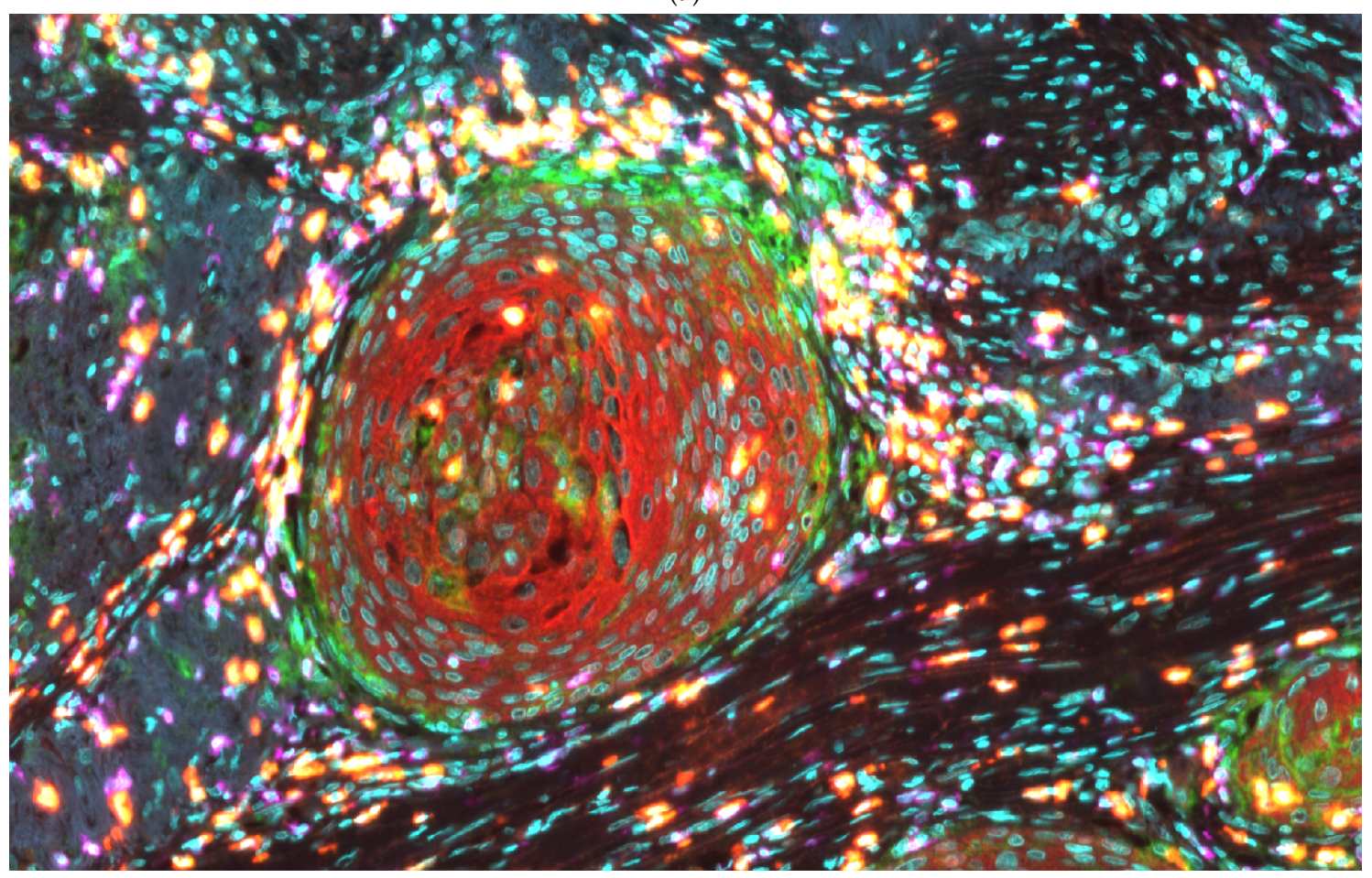

(b)

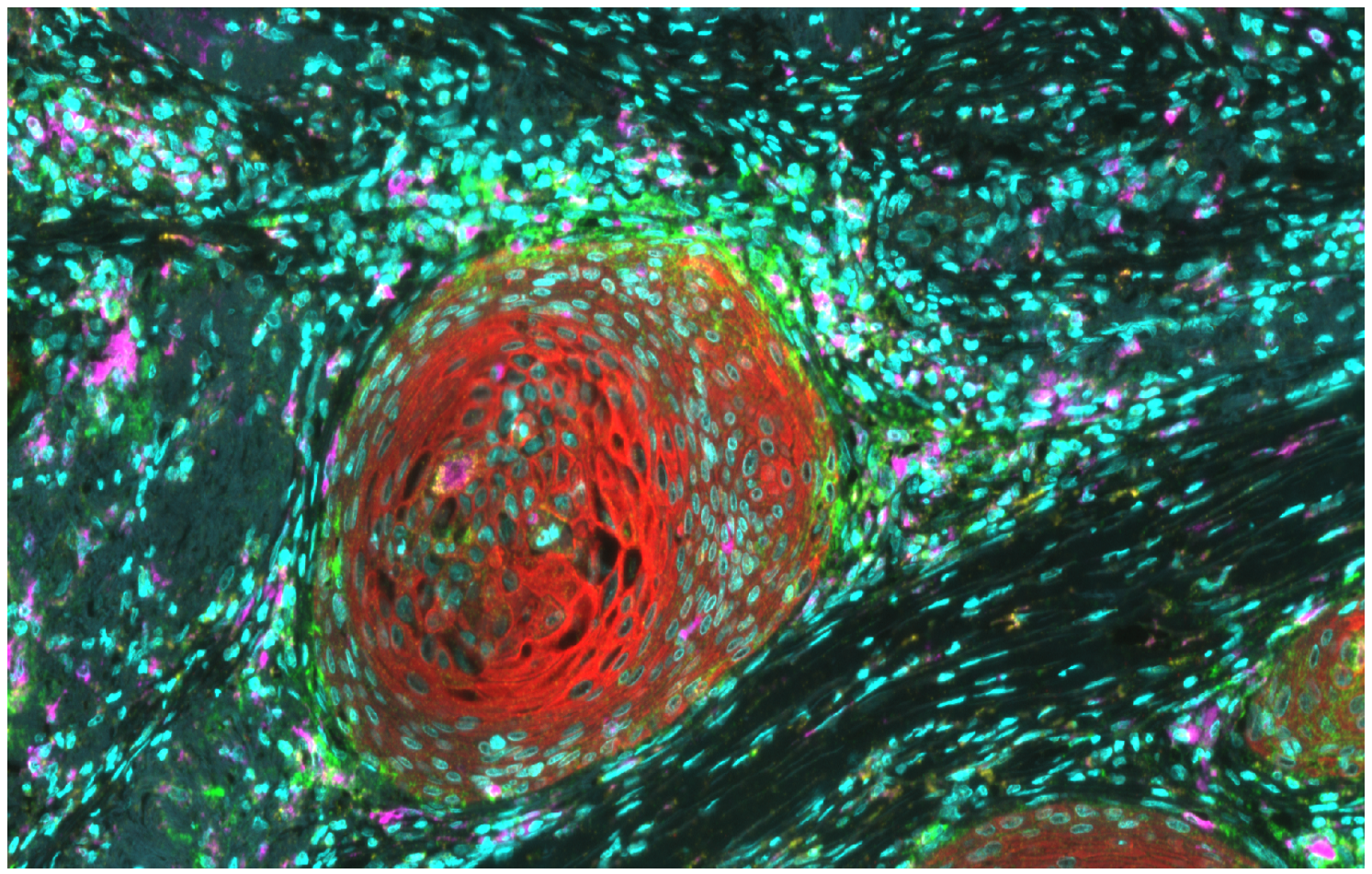

Figure 1. Examples of (a) TILs (Nuclei: Cyan, Cancer: Red, PD-L1: Green, CD3: Purple, CD8: Yellow) and (b) TAMs (Nuclei: Cyan, Cancer: Red, PD-L1: Green, CD68: Purple, CD163: Yellow) visualized using multiplexed immunofluorescence. 


\subsection{Detection of Cell Nuclei}

For nucleus detection, the methodology described by Brieu and Schmidt [39] was adopted. For completeness, a high-level description of this methodology is provided. The methodology is comprised of four distinct stages: (a) A classification random forest (RF) was trained with long-range spatial context features [40] that were extracted from manually annotated foreground and background regions, where a foreground region contained the cell nucleus and vice-versa ( $n=4$, resolution $=1200 \times 1200$ pixels). Subsequently, the RF generated a mask for all immunofluorescence (IF) images [41]. (b) A regression RF was trained to generate proximity maps using coordinates from manual annotations of cell nuclei $(n=750$ from 19 fields of view, resolution $=1485 \times 1485)$. Intuitively, a proximity map enclosed the distance to the closest nucleus center for each pixel of the input image. (c) A regression RF was trained to generate surface area maps using manual annotations ( $n=250$ from 8 fields of view, resolution $=580 \times 580$ pixels) [39]. A surface area model provides a mask of the initial image wherein each pixel is either zero, if it is not a part of a nucleus, or a positive real number, if it is part of a nucleus. The positive real number is the area of the corresponding nucleus. (d) At this stage, nuclei centers (see Supplementary Figure S2) are localized based on the proximity and surface area maps that were generated in (b) and (c) [39]. Note that both the original image and the corresponding mask (produced in (a)) are given as inputs to the models in (b) and (c), using only the PanCK and Hoechst IF channels. Data augmentation with varying scale, rotation, as well as intensity for both PanCK and Hoechst IF channels, was implemented in all stages. Finally, regions with necrotic tissue and any type of artefact, such as autofluorescence, were not included in any of the training data.

\subsection{Segmentation of Epithelial Cells for the Identification of Tumour Buds}

For the quantification of tumours buds, segmentation of the epithelial cells was required. The CNN-RF methodology described by Brieu et al. [42], and extended in [30], was adopted. Briefly, a convolutional neural network (CNN) was trained on an annotated dataset of epithelium and non-epithelium IF images. The dataset comprised $142 \times 142$ pixel regions, selected by an expert. The Hoechst, PanCK, CD3 and CD8 IF channels were normalised following the approach described by Brieu et al. [42]. An intensity interval was imposed on the PD-L1, CD163, and CD68 IF channels by computing the minimum and maximum values from the segmented epithelium regions of each slide. Once trained, the CNN produced a coarse segmentation mask of epithelium regions. Normalized PanCK, Hoechst, CD3, and CD8 channels of the IF images were used as input to the CNN. The predicted epithelium probability layer was used, together with the original IF channels, as input to a RF. Finer-grained segmentation masks were produced by the RF, enabling a more accurate segmentation of the epithelium. As detailed in previous work [30], the output of the CNN-RF is finally ensembled with the output of a semantic segmentation network [43] to generate the final epithelium segmentation results. TBs were classified as epithelium objects containing from one to four nuclei [30]. A total number of 97,262 patches were used for training and 9742 patches for validation of the CNN-RF model. The semantic segmentation network was trained with 19,093 patches and validated with 6987 patches of $256 \times 256$ pixels.

\subsection{Cell Classification}

For cell classification, given the normalised IF channels, a circular neighbourhood of each cell nuclei is defined $(11 \times 11$ pixel radius) and the mean normalised intensity of the neighbourhood is computed for each IF marker (CD3, CD8, CD68, CD163, PD-L1 and PanCK). Cells are classified as positive or negative for a given IF marker if the corresponding mean normalized intensity is above or below a determined threshold, respectively. In our experiments, the threshold for all the IF markers was set to $32 / 256=0.125$. 


\subsection{Pairwise Spatial Distributions of Lymphocytes, Macrophages, Tumour Buds and PD-L1}

The point coordinates of cell nuclei and immune checkpoint ligand PD-L1 expression were localized across the whole slide images (WSIs), as shown in Figure 2. The Ripley's K function [44] was adopted to investigate how TBs, PD-L1, and the different populations of immune cells are distributed around each other. In particular, given two populations $X$ and $Y$, Ripley's K function estimates the density of $Y$ within a circle of radius $r$ around points $X$. As illustrated in Figure 3, assuming a Poisson distribution, the Ripley's K function can identify whether a population $Y$ is dispersed, randomly distributed, or clustered around another population $X$. The $K$ function is given as

$$
K_{x y}(r)=\frac{1}{\lambda_{y}} \mathbb{E}[\text { number of points } y \text { within a distance } r \text { around a point } x]
$$

where $\mathbb{E}[\cdot]$ encloses all of the points of type $y$ within a distance $r$ of a randomly selected point of type $x$ and $\lambda_{y}$ is the number of points $y$ per unit area in the region of interest.

(a)

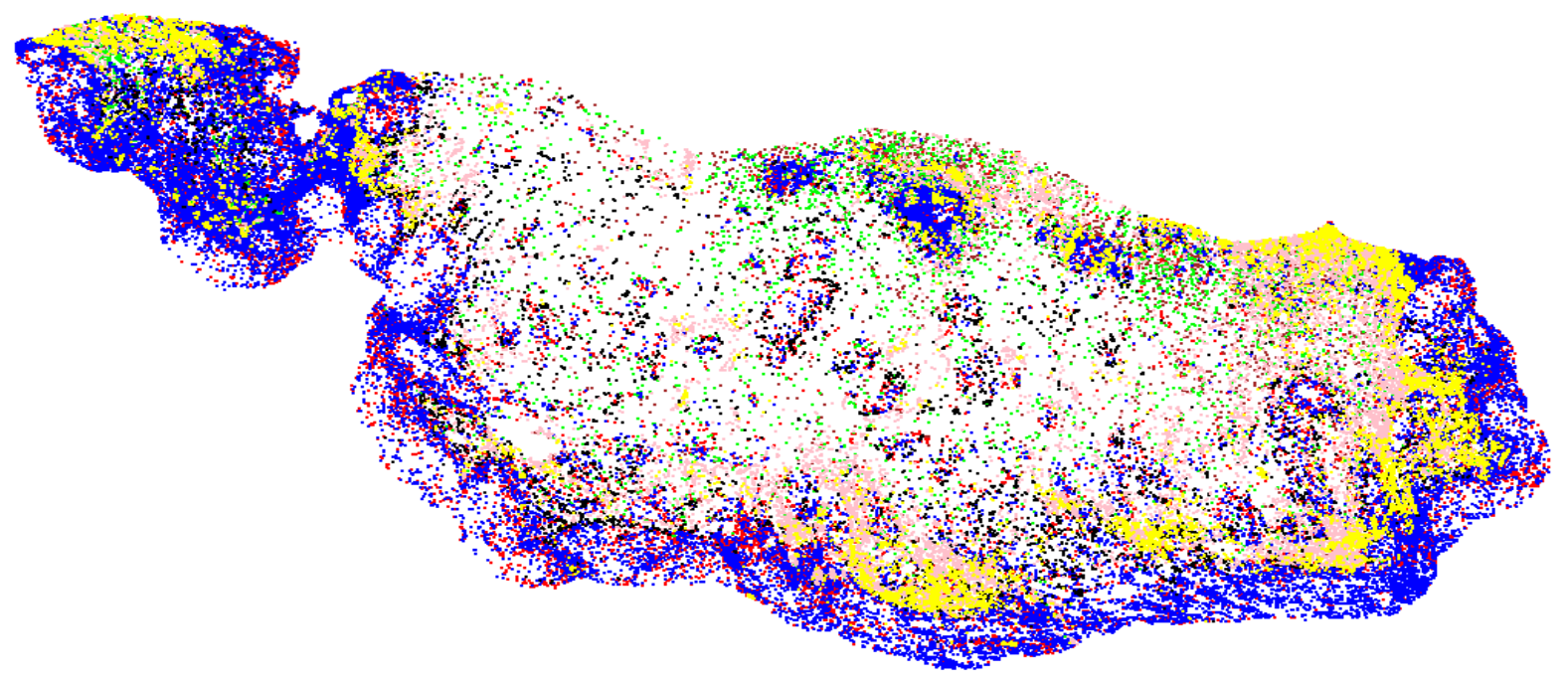

(b)

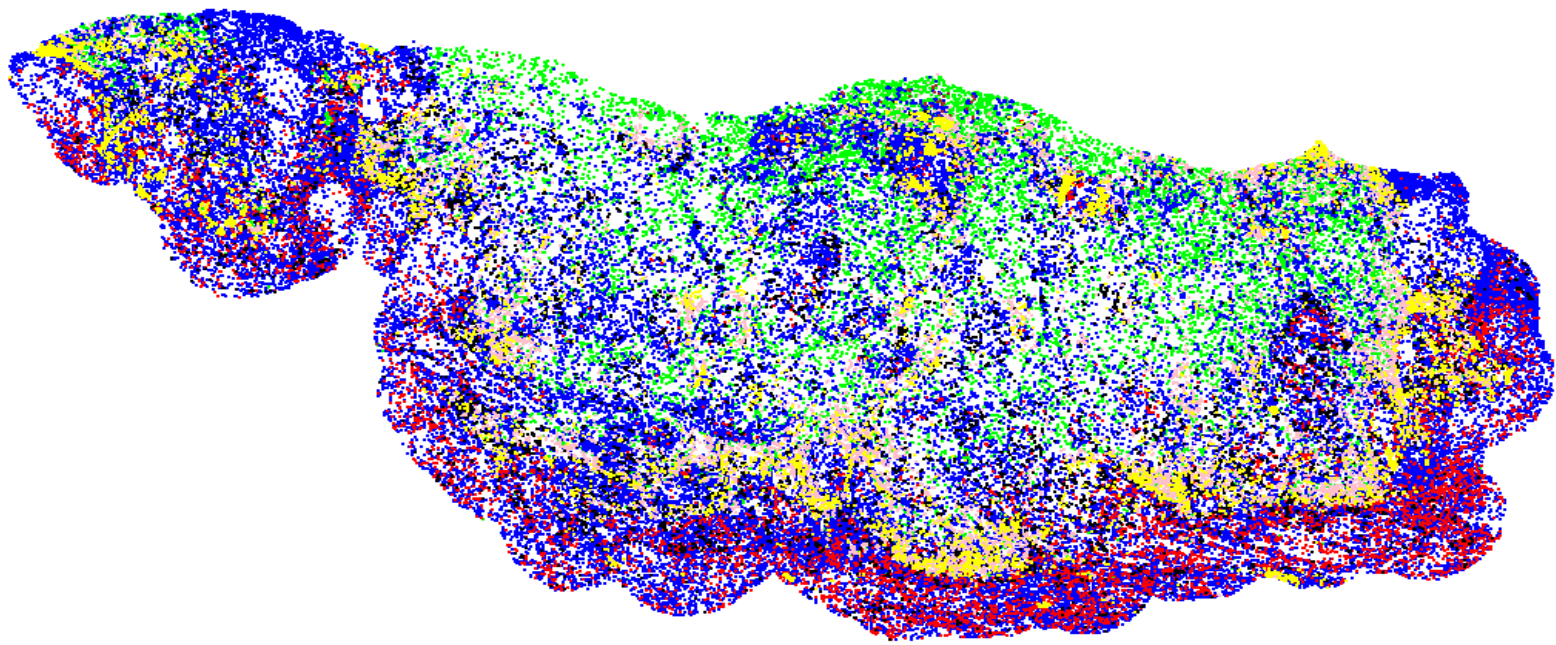

Figure 2. Nuclei localization of (a) TILs $\left(\mathrm{CD}^{+} \mathrm{PanCK}^{+}\right.$: Brown, $\mathrm{CD}^{+} \mathrm{PanCK}^{-}$: Red, $\mathrm{CD}^{+} \mathrm{PanCK}^{+}$: Green, $\mathrm{CD}^{+} \mathrm{PanCK}^{-}$: Blue, TB: black, PD-L1 ${ }^{+}$PanCK ${ }^{+}$: Pink, PD-L1 ${ }^{+}$PanCK $^{-}$: Yellow) and (b) TAMs $\left(\mathrm{CD}_{163}{ }^{+}\right.$PanCK $^{+}$: Brown, $\mathrm{CD} 163^{+} \mathrm{PanCK}^{-}$: Red, $\mathrm{CD}^{+} 8^{+}$PanCK $^{+}$: Green, $\mathrm{CD}^{+} 8^{+} \mathrm{PanCK}^{-}$: Blue, TB: Black, PD-L1 ${ }^{+}$PanCK $^{+}$: Pink, PD-L1 ${ }^{+}$PanCK ${ }^{-}$: Yellow) across the WSI. 
(a)

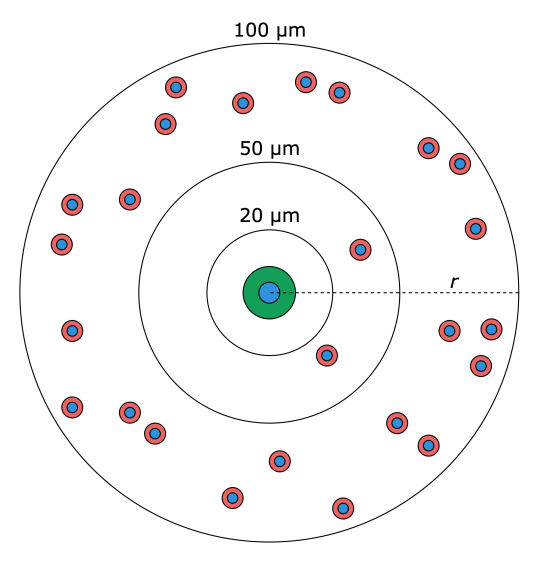

(c)

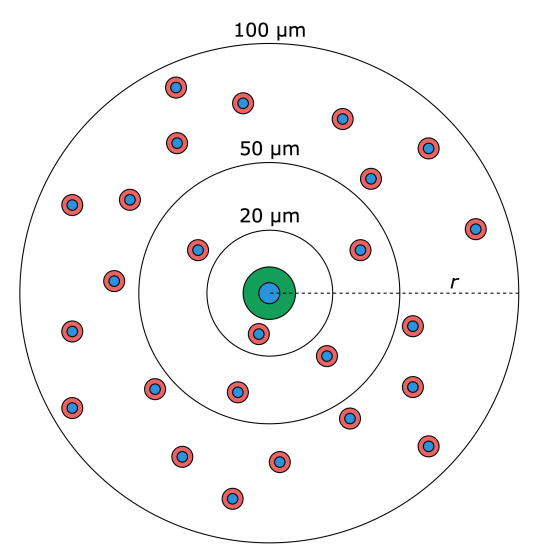

(e)

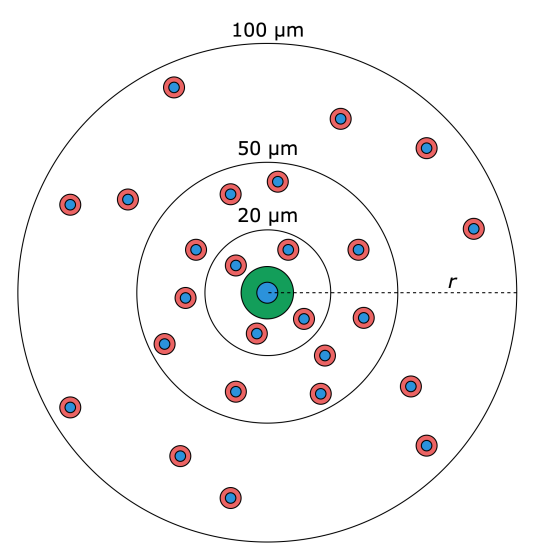

O) tumour buds

O) immune cells (b)

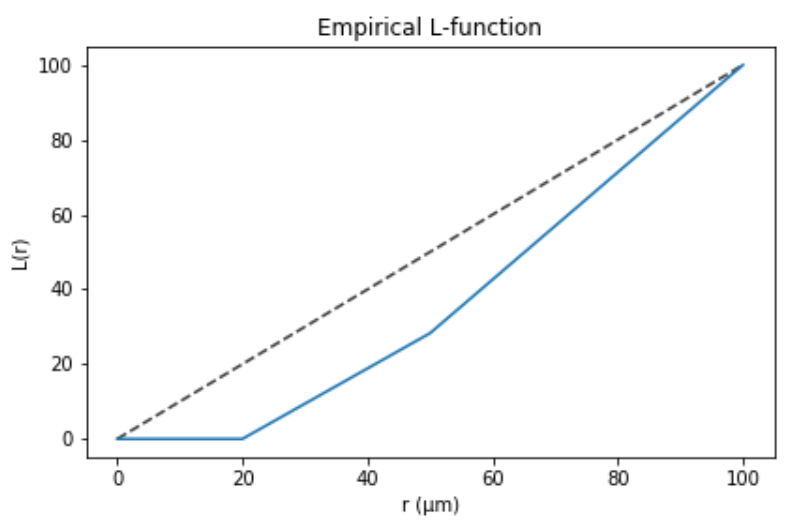

(d)

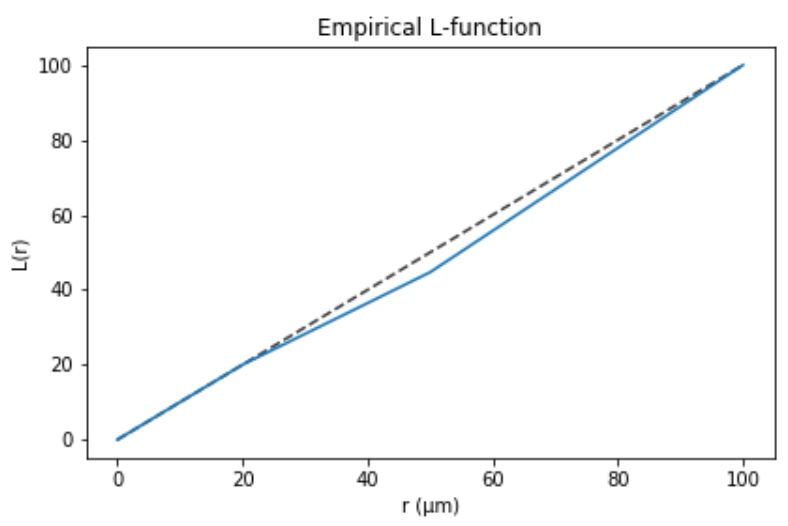

(f)

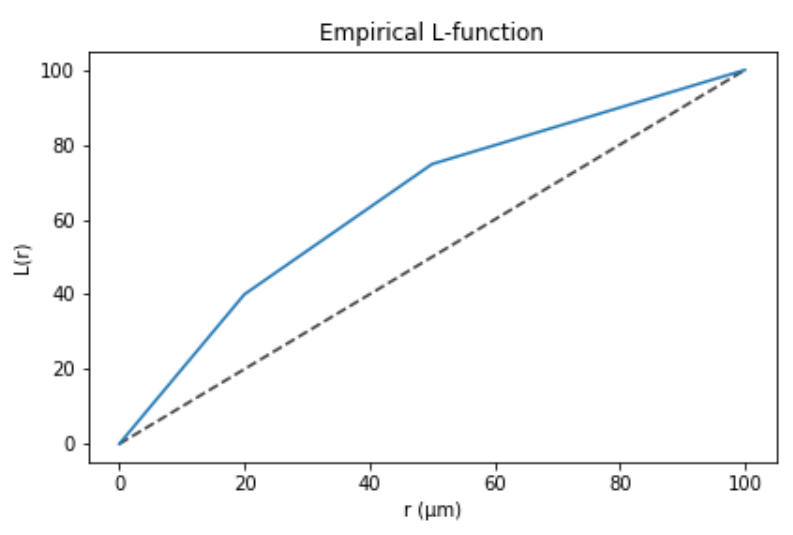

Figure 3. Schematic representation of different immune cell distributions from the nuclear centre of a tumour bud (a,c,e), and their corresponding $L$ function values at different radii $(\mathbf{b}, \mathbf{d}, \mathbf{f})$. The immune cell population is either $(\mathbf{a}, \mathbf{b})$ dispersed, $(\mathbf{c}, \mathbf{d})$ randomly distributed, or $(\mathbf{e}, \mathbf{f})$ clustered around the tumour bud. 
Theoretically, if the point pattern of points $Y$ around $X$ follows complete spatial randomness, also known as a homogeneous Poisson process, the value of $\mathrm{K}$ function is $\pi r^{2}$. The $L$ function [45] is a modification of Equation (1), so that the expected output value is $r$, i.e.,

$$
L_{x y}(r)=\sqrt{\frac{K_{x y}(r)}{\pi}}
$$

This enables a more intuitive interpretation of the function's output value in relation to $r$. The $L$ function was calculated for TAMs, TILs, and PD-L1 surrounding TBs, as well as the PD-L1 surrounding TAMs and TILs, for a series of increasing distances $r$, where $r \in\{20,50,100,150,200,250\} \mu \mathrm{m}$. While some approaches calculate the area under the curve of the $L$ function against different $r$ values [46], we provided the pairwise spatial distributions between PD-L1, TBs, and the immune populations, directly to the ML classifiers as distinct features.

\subsection{Binary Survival Analysis}

Survival analysis is broadly defined as the analysis of data that involve the time to the occurrence of an event of interest [47]. Herein, the event of interest was the death of an individual due to MIBC. A characteristic of survival analysis is censoring. In our cohort, some patients were right-censored, either because the end of study was reached and the event of interest did not occur, or because the patients succumbed to a cause other than MIBC (abbreviated as OTD-censoring) [47].

Patient survivability was binarized based on a specific time cut-off. Similar to previous works, a 5 year prognosis was investigated $[36,48,49]$. Patients that succumbed to MIBC within 5 years were denoted as patients with a bad prognosis, whereas those that survived the 5-year cut-off were denoted as patients with a good prognosis. Inevitably, patients that died due to an unrelated cause prior to the prognostic cut-off, i.e., they were part of the OTD-censored data, had to be excluded (19\% patient exclusion). It is worth mentioning that removing these patients does not introduce bias, since time to censoring was random, i.e., OTD-censoring was not known a priori. A consequence of this approach is that survival analysis was turned into a binary classification problem. Furthermore, due to the removal of censoring, traditional ML models were readily employable.

\subsection{Model Selection, Algorithm Selection, and Performance Evaluation}

Both model selection and algorithm selection attempt to collectively maximize the predictive performance of the final ML model. However, ML algorithms are prone to overfitting, i.e., in finding and using patterns which arise from noise in the data. Such noisy patterns do not generally extend beyond the specific dataset, since noise is typically random. With both a small dataset and a complicated model, the likelihood of overfitting increases. Testing the performance of a trained ML model on unseen data constitutes the mainstay of evaluating the generalizability of an ML model and, therefore, in identifying whether a model has overfitted. As such, a subset of the initial cohort was kept aside as the testing dataset. In particular, using stratified random sampling, two subsets were created: the training set with $75 \%$ of the initial data (58 patients), and the testing set with $25 \%$ (20 patients). The testing set was only used at the performance evaluation stage to avoid introducing bias to the generalization performance estimate.

Traditionally, model selection is the process by which a ML algorithm is configured. Most ML algorithms come with a number of configuration variables, commonly referred to as hyperparameters. Even though common hyperparameter configurations can be employed, it has been observed that hyperparameter tuning for a specific task can be the key between chance and state-of-the-art models [50]. Since manual tuning can be timeconsuming and counter-intuitive in high-dimensional spaces, most ML methodologies adopt automated hyperparameter tuning. 
One of the most popular approaches in model selection constitutes a grid search, where each hyperparameter is given a predefined list of values, and the best hyperparameter configuration is selected after evaluating all the combinations. For example, given hyperparameters $A$ and $B$, and lists $V_{A}=[1,10,100]$ and $V_{B}=[0.1,0.5]$, the following combinations were evaluated under grid search: $\forall(A, B) \in[(1,0.1),(1,0.5),(10,0.1),(10,0.5)$, $(100,0.1),(100,0.5)]$. However, as shown by Bergstra and Bengio [51], random sampling provides a better tuning strategy.

In random search, a number of hyperparameter configurations are evaluated by sampling from predefined hyperparameter distributions and densities. For example, given hyperparameters $A$ and $B$ with $D_{A} \sim N(0,1)$ and $V_{B}=[0.1,0.5]$, where $D_{A}$ is a standard Gaussian distribution, the following five combinations could have been sampled and evaluated under a random search: $\forall(A, B) \in[(-0.12,0.1),(-0.14,0.5),(-0.94,0.5)$, $(0.44,0.1),(-1.3,0.5)]$. In our methodology, 200 hyperparameter configurations were randomly sampled and evaluated for each ML algorithm. A table of the distributions and densities used is provided in Supplementary Table S4.

Not every ML algorithm will perform equally well in different problems and with different data. In addition, there is no theoretical ranking suggesting that one algorithm is better than another [52]. Hence, similar to hyperparameter tuning, algorithm selection is yet another meta-optimization task that needs to be performed for maximizing predictive performance. However, as argued by Bergstra et al. [50]: "Since the performance of a given technique depends on both the fundamental quality of the algorithm and the details of its tuning, it is sometimes difficult to know whether a given technique is genuinely better, or simply better tuned." Consequently, algorithm selection should involve model selection. Therefore, each ML algorithm was first tuned using five-fold cross-validation and then compared against each other using two-fold cross-validation. This nested cross-validation translates to optimization of the hyperparameters of each ML algorithm twice, and then measurement of their performance on the corresponding evaluation folds (see Figure 4). Subsequently, the ML algorithm which performed better than the others across two different training and validation folds is selected. It is important to highlight how, in most cases, each ML algorithm is evaluated based on two different hyperparameter configurations. Nevertheless, once the ML algorithm was selected, yet another hyperparameter-tuning phase is implemented to find an optimal hyperparameter configuration based on the whole training dataset. Five ML algorithms with different theoretical underpinnings were selected to compete against each other: decision tree (DT), RF, support vector machine (SVM), linear regression (LR), and $k$ nearest neighbours (KNN). Finally, a preprocessing step of feature normalisation was added to all classifiers, except DT and RF.

\subsection{Stratified Sampling}

In order to avoid sampling subsets with different class distributions (classes are based on survival with a 5-year cut-off) to the original cohort, stratified sampling was used. Intuitively, when sampling from a dataset with stratification, proportionally, many patients from each class were sampled.- as an example, given a dataset with 75 patients of class $C_{1}$ and 25 patients of class $C_{2}$, a $20 \%$ sample would contain 15 patients from class $C_{1}$ and 5 patients from class $C_{2}$. 


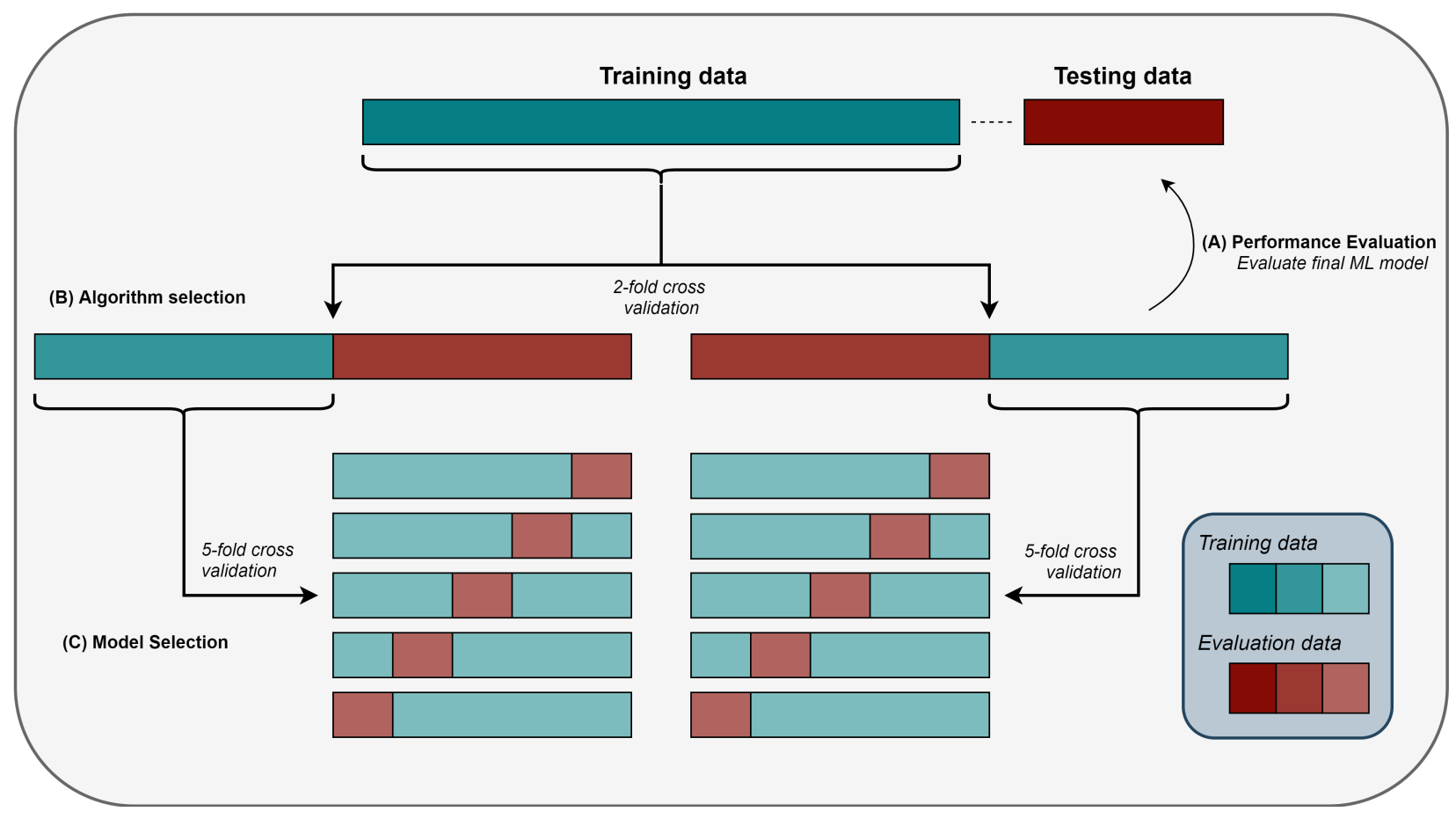

Figure 4. Pictorial representation of nested cross-validation with an independent testing set. (A) Performance Evaluation: The best ML algorithm (selected by the outer cross-validation; see (B)) was trained on the training dataset and subsequently evaluated on the testing dataset. (B) Algorithm Selection: Each ML algorithm (with hyperparameters tuned based on the inner cross-validation; see (C)) was trained and tested on the corresponding training and evaluation folds, respectively. The best ML algorithm was selected based on the average performance of both evaluation folds. (C) Model Selection: ML models with randomly sampled hyperparameter configurations were trained and tested based on a five-fold crossvalidation. The best hyperparameter configuration for each ML algorithm was selected based on the performance on all five evaluation folds.

\section{Results}

\subsection{Patient Characteristics}

A total of 78 patients diagnosed with MIBC were included in this study. The median age of the patients was 68 years (range 29-87 years), with 43 males and 35 females. According to the TNM staging system guidelines [8], our cohort consists of 17 stage II, 29 stage IIIA, 5 stage IIIB, and 27 stage IV patients. Twenty-seven patients had distant metastasis at time of surgery. No positive lymph nodes were found in 57 patients, and 1-2 lymph nodes contained tumour cells in 21 patients. Of the 78 patients, 53 patients died due to bladder cancer. Follow-up information was available for all the patients (range 1-113 months). The clinicopathological characteristics of the cohort are summarised in Table 1.

\subsection{Fully Automated Feature Extraction}

The entire FFPE tissue section of each MIBC patient was digitized into a WSI, encompassing both muscle-invasive urothelial carcinoma and adjacent benign tissue. Multiplex immunofluorescence, using TSA, enabled the detection of TILs (general CD3 and cytotoxic CD8 T-cells), TAMs (total CD68 macrophages and M2 CD163 macrophages), PD-L1 ${ }^{+}$cells, cell nuclei (Hoechst), and epithelial cancer cells (Pancytokeratin) including TBs across the WSI of each patient. Machine-learning-based image analysis allowed for the localization of each cell, which was subsequently classified depending on its IF signal as either a: (1) TB, (2) M1 macrophage, (3) M2 macrophage, (4) total macrophage, (5) general T cell, (6) cytotoxic $\mathrm{T}$ cell, or (7) PD-L1 ${ }^{+}$cell. Based on the above seven classes, a total of 186 quantitative features were extracted from the tumour core and invasive front of each WSI, including the number and density of different cell types, the total size of tumour areas, and the pairwise 
spatial distributions between immune and cancer cells. The tumour core is defined as the main tumour mass and the invasive front as the border of the tumour core, with a width of $1000 \mu \mathrm{m}$ (500 $\mu \mathrm{m}$ inside and $500 \mu \mathrm{m}$ outside of the border, defining the invasive frontin and frontout, respectively), as shown in Figure 5. Feature extraction was performed using Definiens Tissue Phenomics ${ }^{\circledR}$ software (Definiens AG, Munich, Germany) [53-55]. A list of all extracted features is provided in Supplementary Table S11.

Table 1. Patient cohort characteristics.

\begin{tabular}{cc}
\hline Characteristics & Summary \\
\hline MIBC patients & $N=78$ \\
Median survival (range) & $19(1-113)$ months \\
Age & $66 \pm 11$ years \\
Gender & $55 \%$ Male; $45 \%$ Female \\
TNM stage & $17(22 \%)$ \\
II & $29(37 \%)$ \\
IIIA & $5(6 \%)$ \\
IIIB & $27(35 \%)$ \\
IV & \\
Tumour (T) & $18(23 \%)$ \\
T2 & $39(50 \%)$ \\
T3 & $21(27 \%)$ \\
T4 & \\
Node (N) & $57(73 \%)$ \\
N0 & $13(17 \%)$ \\
N1 & $8(10 \%)$ \\
N2 & \\
Metastasis (M) & $51(65 \%)$ \\
M0 & $27(35 \%)$ \\
M1 &
\end{tabular}

(a)

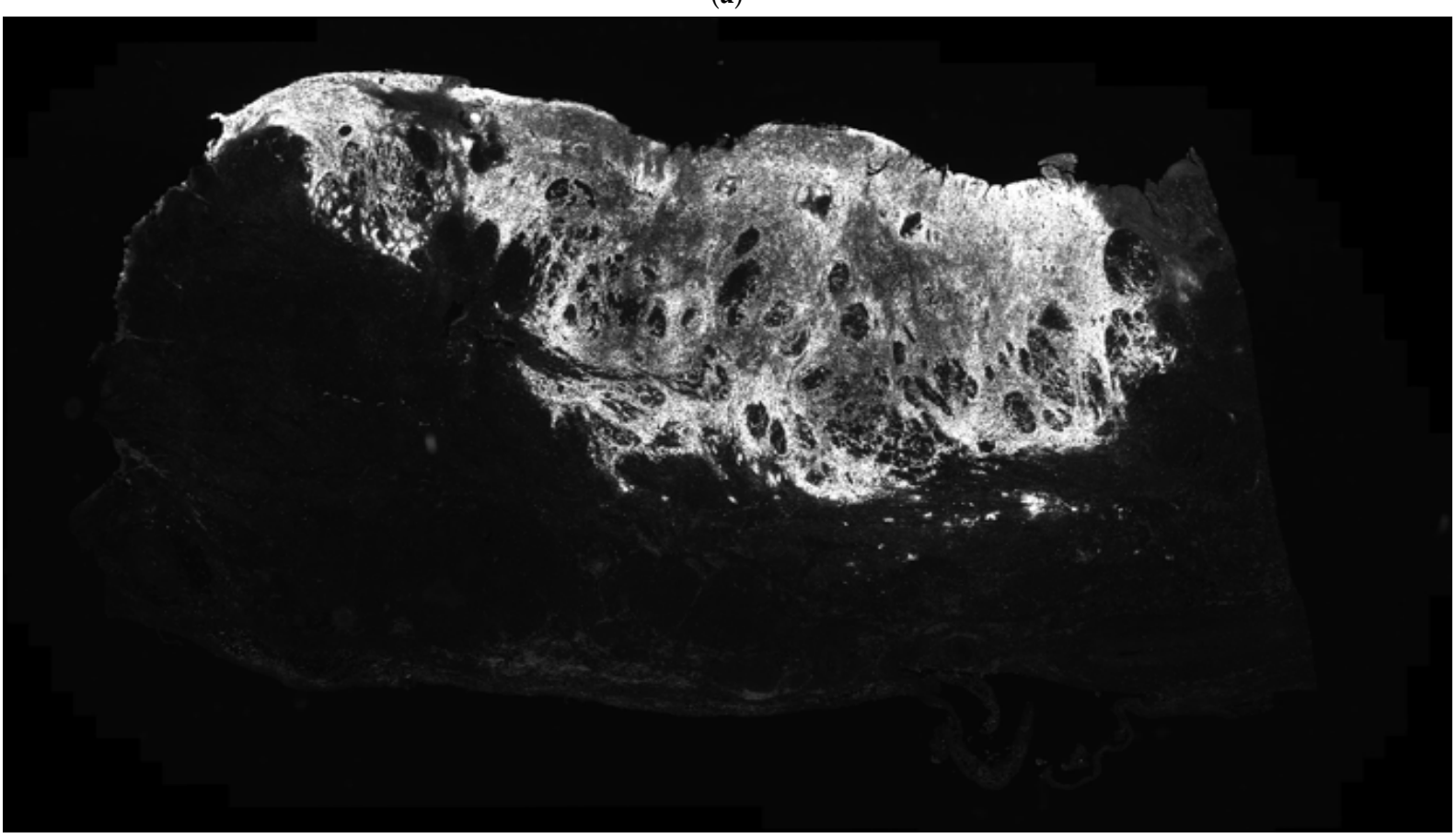

Figure 5. Cont. 
(b)

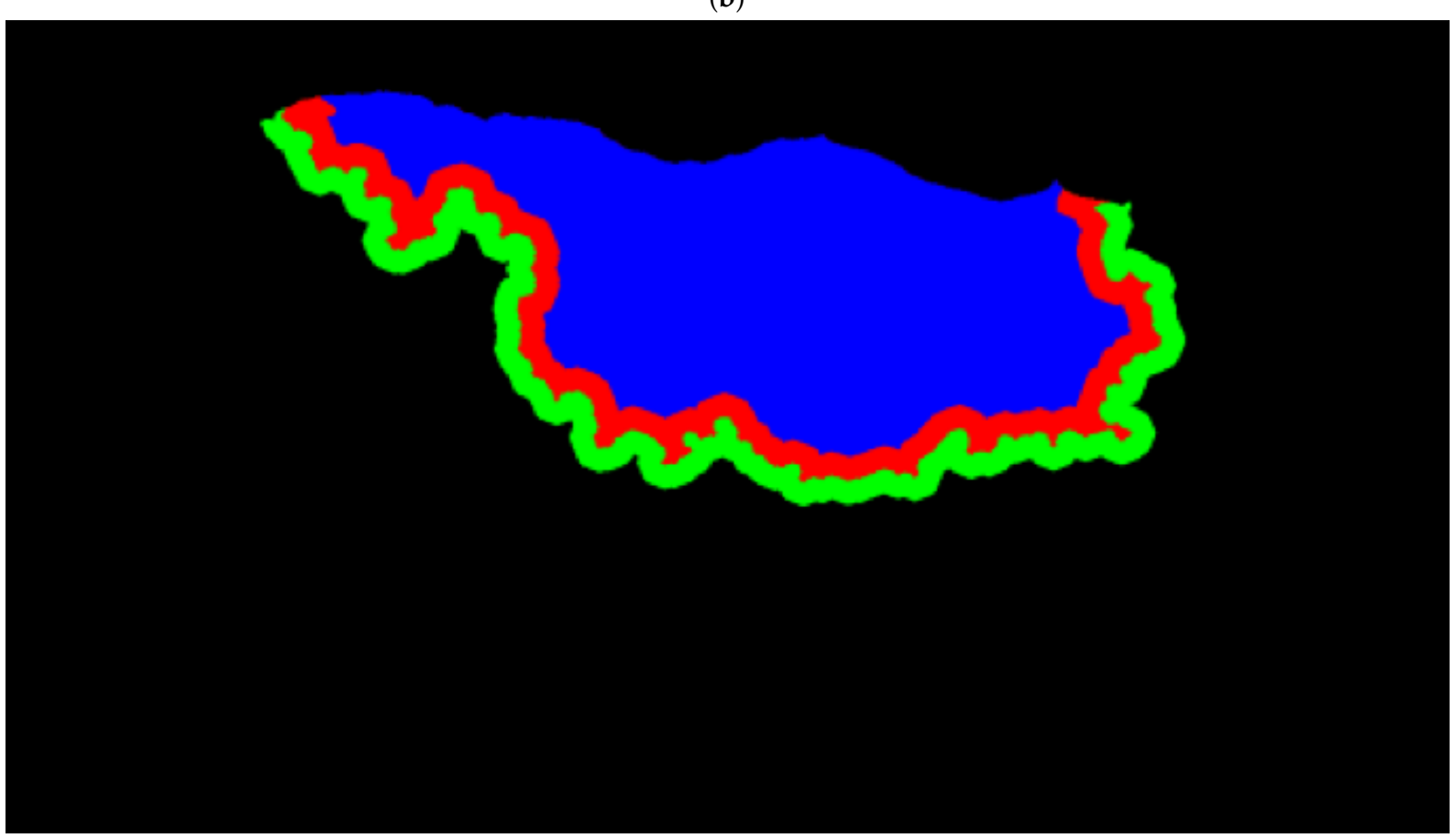

Figure 5. (a) Whole-slide immunofluorescence image based on the PanCK channel, (b) Segmentation of the corresponding tissue (a) into tumour core (blue), invasive frontin (red) and frontout (green) using the PanCK channel.

\subsection{Feature Space and Feature Selection}

In order to capture multiple aspects of the disease, features from both clinical reports and whole-slide immunofluorescence images were quantified. Herein, the number and density of PD-L1-positive and -negative immune cell populations, as well as of TBs from the WSIs are labelled as "image features". The pairwise spatial distributions between immune cells and TBs are termed "spatial features". Finally, clinicopathological features such as age, gender, and TNM stage are termed "clinical features". Information regarding the administration of chemotherapy before or after cystectomy (neoadjuvant or adjuvant) was not provided in a sufficient degree of detail in the authorised release of deidentified clinical records. To maintain the reproducibility of our methodology, chemotherapy was not included as a clinical feature. Altogether, 201 features were quantified-126 image, 60 spatial, and 15 clinical features (the complete feature list is given in Supplementary Table S11). To investigate whether smaller feature spaces result in better ML models, we ran the same ML workflow over different feature sets. In particular, our experiments were based on the following seven feature sets: (i) image, (ii) spatial, (iii) clinical, (iv) image and spatial, (v) image and clinical, (vi) spatial and clinical, (vii) image, spatial, and clinical.

\subsection{Machine Learning Models and Optimizing Metric}

Five ML algorithms with different theoretical underpinnings were selected to investigate whether the extracted features could predict 5-year survivability in MIBC patients; DT, RF, SVM, LR, and KNN. The optimizing metric throughout experimentation was the area under the receiver operating characteristic (AUROC). At the final evaluation phase, classification accuracy, sensitivity, specificity, F1 score, and hazard ratios were also computed for ease of comparative analysis, as shown in Table 2. In order to compute the aforementioned metrics, the optimal threshold values were automatically selected at the final stage based on the training set performance. Hazard ratios and the associated confidence intervals were calculated using univariate Cox regression. 
Table 2. Comparison between our ensemble model and TNM staging. The better performance between the ensemble model and TNM staging for each evaluation metric is shown in bold. * $95 \%$ Confidence Interval.

\begin{tabular}{ccccc}
\hline & \multicolumn{2}{c}{ Training Set } & \multicolumn{2}{c}{ Testing Set } \\
\hline $\begin{array}{c}\text { Evaluation } \\
\text { Metrics }\end{array}$ & $\begin{array}{c}\text { Ensemble } \\
\text { Model }\end{array}$ & TNM Staging & $\begin{array}{c}\text { Ensemble } \\
\text { Model }\end{array}$ & TNM Staging \\
\hline AUROC & $\mathbf{9 8 . 3}$ & 71.6 & $\mathbf{8 9 . 3}$ & 64.3 \\
Accuracy & $\mathbf{9 4 . 8}$ & 65.5 & $\mathbf{8 0 . 0}$ & 50.0 \\
Sensitivity & $\mathbf{8 9 . 5}$ & $\mathbf{8 9 . 5}$ & $\mathbf{1 0 0 . 0}$ & $\mathbf{1 0 0 . 0}$ \\
Specificity & $\mathbf{9 7 . 4}$ & 53.8 & $\mathbf{7 1 . 4}$ & 28.6 \\
F1 score & $\mathbf{8 3 . 3}$ & 67.7 & $\mathbf{8 3 . 3}$ & 44.0 \\
Hazard ratio & $\mathbf{4 5 . 9}$ & 4.4 & $\mathbf{3 2 . 5}$ & 3.3 \\
& $(\mathbf{6 . 2 , 3 4 1 . 1 ) *}$ & $(2.3,8.6)^{*}$ & $(\mathbf{3 . 9 , 2 7 0 . 3 )}$ & $(1.0,11.0)^{*}$ \\
\hline
\end{tabular}

\subsection{Proposed Ensemble Model}

Nested cross validation was implemented to avoid overfitting while maximizing the predictive performance of ML classifiers. In addition, a separate test set was held aside to estimate the generalization performance of the final classifier.

For each of the tested feature sets, the classifier with the highest average AUROC was selected. In case of similar average AUROC between two ML classifiers using the same feature set, we selected the one exhibiting the least variance. The results are shown in Supplementary Table S5. Since multiple classifiers exhibited a similar performance across the different feature sets, instead of employing a single classifier, we combined the best ones into an ensemble model. In particular, our ensemble model consists of a linear support vector machine (LSVM) that uses image features (72.8 \pm 0.3 AUROC), a DT that uses image and clinical features (68.8 \pm 0.8 AUROC), an LR that uses image and spatial features (70.2 \pm 14.7 AUROC), and an RF that uses all features (67.3 \pm 5.8 AUROC). Following hyperparameter tuning for each one of the selected classifiers on the whole training set, without cross-validation, our ensemble model was evaluated on the independent testing set, achieving $89.3 \%$ AUROC and a highly significant separation of patients into low- and high-risk groups $\left(p\right.$ value $\left.=7 \times 10^{-6}\right)$. Patients were classified as high-risk by the ensemble model if two or more of the submodels predicted a bad prognosis.

\subsection{Pessimistic Bias}

The large difference between the generalization estimates of algorithm selection and performance evaluation (see Tables S1 and 2) can be mostly attributed to pessimistic bias [56]. Given the already small dataset, withholding half of the training dataset for evaluation, due to two-fold cross-validation, increases the chance that an ML model will underfit, i.e., its maximum representation capacity will not be reached [56]. Therefore, the generalization estimate from performance evaluation (Table 2) is more reliable, since the whole training set was used.

\subsection{Comparing against TNM Staging}

In order to compare this against the gold standard in clinical practice, TNM, patients had to be stratified into low- and high-risk groups. Based on a pairwise log-rank test comparison in the training dataset (results shown in Supplementary Tables S6-S8), stage II and III patients were considered as the low-risk group, whereas stage IV patients were considered as the high-risk group. The Kaplan-Meier and ROC curves of TNM staging and our ensemble model on the testing set are shown in Figure 6. To allow further comparative analysis, Kaplan-Meier curves of other clinicopathological features, such as age and gender, are shown in Supplementary Figure S3. In addition, the Kaplan-Meier and ROC curves of each submodel of the ensemble model are shown in Supplementary Figure S4. 
(a)

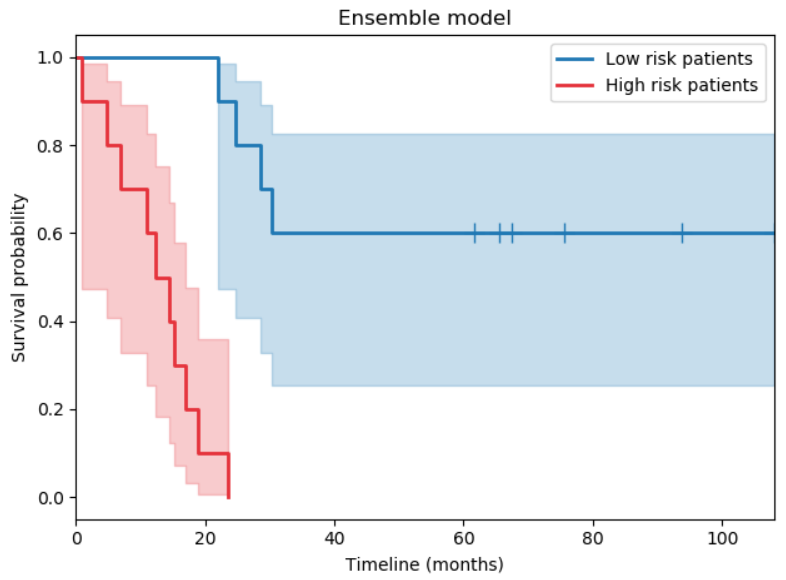

(c)

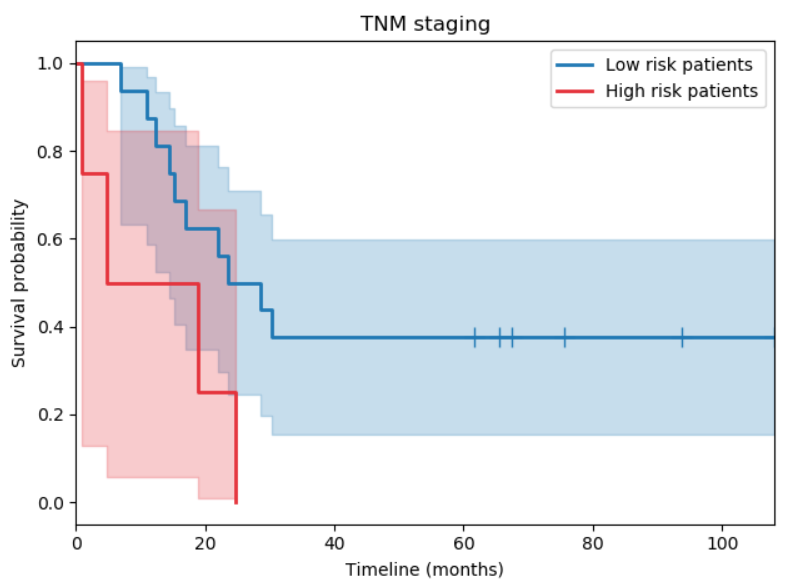

(b)

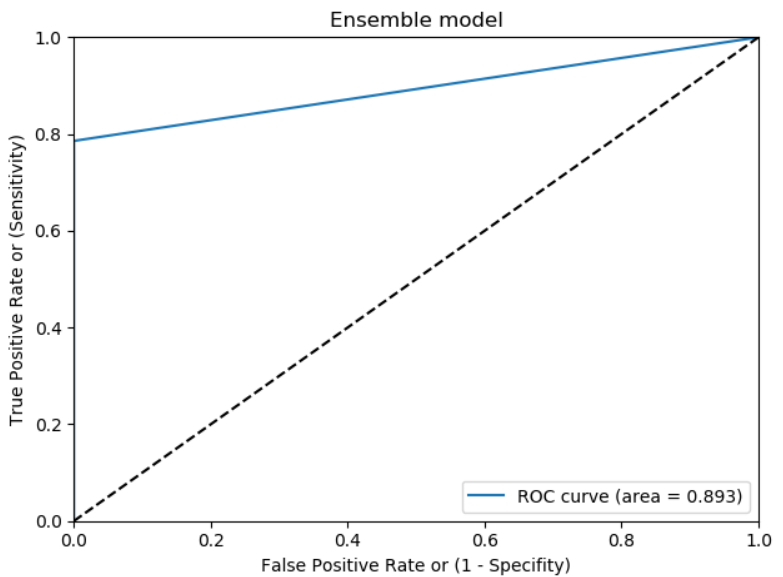

(d)

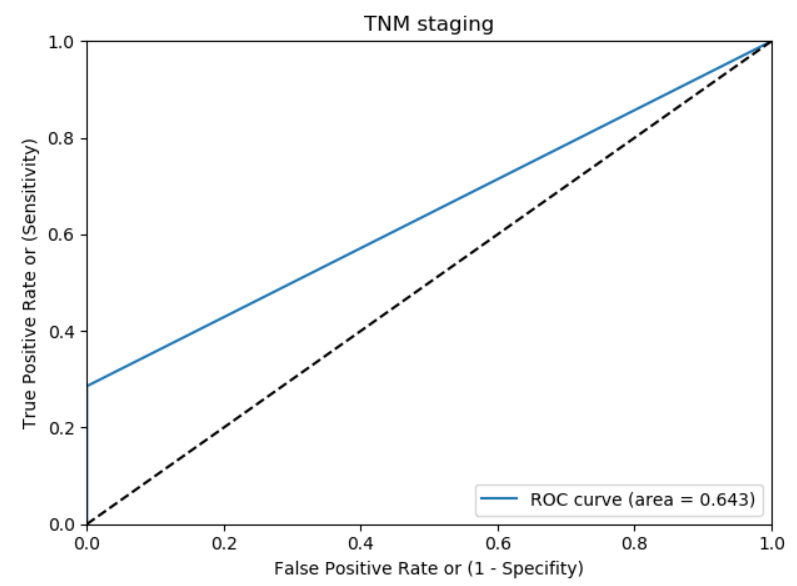

Figure 6. Kaplan-Meier and ROC curves on the testing set for our ensemble model and TNM. Separation was significant based on the (a) ensemble model ( $p$ value $\left.=7 \times 10^{-6}, N_{\text {LowRisk }}=10 \& N_{\text {HighRisk }}=10\right)$ and (c) TNM ( $p$ value $=0.04$, $\left.N_{\text {LowRisk }}=16 \& N_{\text {HighRisk }}=4\right)$. A better AUROC was achieved by $(\mathbf{b})$ the ensemble model $(A U R O C=0.893)$ than $(\mathbf{d})$ the $\operatorname{TNM}(A U R O C=0.643)$.

\subsection{Post-hoc Analysis of Features}

For each classifier of the ensemble model, post-hoc analysis was conducted to reveal the features guiding the survivability prediction. The feature considered at each node of a DT is readily interpretable (see Supplementary Figure S5). For the LR, its coefficients determine the importance, as well as the positive or negative effect, of each feature in patient prognosis. The mean decrease in the Gini index was calculated for each feature of the RF, based on the underlying decision trees [57]. Finally, since the selected SVM had a linear kernel, feature-ranking coefficients were readily available [58]. A threshold was set to filter out features with low feature importance. In particular, the threshold was set to two times the mean importance of all features for the DT, LR, and RF, whereas two times the median importance was used for the LSVM. There were 8, 10, 25, and 16 important features for DT, LR, RF, LSVM, respectively, which are listed in Supplementary Tables S9 and S10. For completeness, Supplementary Tables S12-S14 provide the feature importance values of all features used by LR, RF, and LSVM prior to thresholding. A visualization of the number of intersecting features between the various submodels of our ensemble model is shown in Supplementary Figure S6. 
For the LR and LSVM submodels, the high density of TBs in both the invasive frontin and tumour core is highlighted as an indicator of bad prognosis. On the contrary, a high density of $\mathrm{CD}^{+}, \mathrm{CD}^{+}$and $\mathrm{CD}^{+} 8^{+}$cells is consistently identified as a marker of good prognosis. In addition, a high number of $\mathrm{CD}^{+}, \mathrm{CD}^{+} 8^{+} \mathrm{PD}-\mathrm{L}^{+}{ }^{+}$and $\mathrm{CD} 163^{+} \mathrm{PD}-\mathrm{L} 1^{+}$cells in the invasive front, as well as the presence of $\mathrm{CD}^{+}$cells within a distance of $20 \mu \mathrm{m}$ from TBs, are associated with good prognosis.

For the DT submodel, a low density of $\mathrm{CD} 8^{+}$, high $\mathrm{PD}-\mathrm{L}^{+}$expression, and high number of TBs (all in frontout) lead to bad prognosis, whereas, given a low density of $\mathrm{CD} 68^{+}$and PD-L1 ${ }^{+}$expression in frontout, the prognosis depends on the number of $\mathrm{CD}^{+} 8^{+}$in frontin. Finally, the majority of the patients with good prognosis had high $\mathrm{CD}^{+} 8^{+}$in frontout, nonzero $\mathrm{PD}_{\mathrm{L}} 1^{+}$expression in core, low $\mathrm{CD} 163^{+}$in frontout, and high $\mathrm{CD}^{+}$in frontout.

Similar to the previous submodels, TBs and $\mathrm{CD}^{+} 8^{+}$cells were the most important predictors of 5-year prognosis for RF. In addition, RF employed more spatial features than any of the other submodels, including, but not limited to, PD-L1 $1^{+}$expression within a distance of $20 \mu \mathrm{m}$ from TBs and $150 \mu \mathrm{m}$ from M2 macrophages and the presence of $\mathrm{CD}^{+}$ and $\mathrm{CD} 8^{+}$cells within a distance range of $20-50 \mu \mathrm{m}$ from TB.

\section{Discussion}

In the last decade, advances in the rapidly growing field of tumour immunology have provided further insights into the dynamic nature of the multifaceted immune response throughout the various stages of cancer initiation, evasion, and progression. Concomitantly, multiple research groups have successfully leveraged this new knowledge to improve cancer prognosis, thereby providing evidence for the clinical relevance of immunooncology [11]. Accurate patient prognosis is crucial for improving the survival rates of cancer patients, since it is a prerequisite to delivering the most effective treatment for each patient. In fact, multiple papers have shown that the quantitative characterization of the tumour-immune microenvironment components, including TILs, TAMs, and immune checkpoints, can yield information of prognostic relevance [59-61]. Particularly, tumour cells surrounded by a large number of prominent intra-tumoural and peri-tumoural TILs and M1 macrophages have been related to better prognosis in several types of cancer [62], whereas a high content of M2 macrophages and TBs has been associated with poorer outcome [60]. In addition, related research has reported the significance of PD-L1 expression on tumour tissues as an independent poor prognostic factor [63]. In this paper, we have investigated, for the first time, the prognostic relevance of immune system biomarkers, TILs, TAMs, TBs, and PD-L1, across whole slide immunofluorescence images of MIBC patients.

$H \& E$ is still the most important and commonly used histochemical staining method for studying and diagnosing tissue diseases in histopathology. However, the imaging of H\&E stained FFPE tissue has limitations, including the inability to quantify the complex cellular states as well as identify distinct cell populations in the tumour-immune microenvironment. With the advent of whole-slide imaging and the increasing adoption of digital pathology in the clinic [64], multiplex methodologies have the potential to provide significantly more information about the underlying tumour-immune microenvironment than single-marker (i.e., single-label immunohistochemistry) and conventional histochemicalstaining-based methodologies [65]. The development of single-protein-based biomarkers to explain patient-level behaviour is hindered by the vast signalling network mediating the heterotypic cell-cell crosstalk between cancer, stromal and immune cells. Instead, with multiplexed methodologies, various proteins can be simultaneously captured on a single tissue sample, encapsulating the tumour-immune architecture from the cellular level down to the subcellular, and ultimately providing more information about the microenvironment.

In our approach, multiplexed immunofluorescence was used to visualize TBs, general and cytotoxic T-cells, M1, M2, and total macrophages, and their co-expression of immune checkpoint ligand PD-L1, in order to quantify their numbers and densities, as well as their pairwise spatial distributions across defined areas (tumour core, invasive frontin and frontout) within a WSI. In the last decade, multiple studies have investigated the topo- 
graphical distribution of the immune cells within the tumour microenvironment $[66,67]$. It is known that tumour-infiltrating immune cells are scattered in the tumour core and the invasive front, whereas their density in each tumour region is correlated with patient outcome [68]. Furthermore, the analysis of multiple tumour regions (tumour core and invasive front) was shown to improve the prediction accuracy of patient survival compared to single-region analysis $[10,68]$. In addition, Immunoscore, a classification system based on the quantification of two lymphocyte populations (CD3 and CD8) within the tumour core and the invasive front of tumour, has been shown to have a prognostic significance superior to that of the TNM staging system in patients with colorectal carcinoma [10,69]. The image, spatial, and clinical features contain a large amount of information about the state of the disease, and collectively portray a more holistic view of each patient's pathophysiology. We hypothesized that these features can predict the aggressiveness of MIBC, and therefore suggest whether a patient should be considered at low or high risk of disease-specific death.

ML contains a plethora of classifiers that have been employed with success in multiple instances, including diagnosis, segmentation, prognosis, and even therapy planning [35]. In our methodology, survival analysis is turned into a binary classification problem, thus enabling traditional ML algorithms and workflows to be readily employable. In addition, to counter the possibility of overfitting due to having a small dataset and high-dimensional feature space, nested cross-validation with a separate testing set was adopted. The proposed ensemble model significantly surpasses all metrics-AUROC, Accuracy, Specificity, F1 score, Hazard ratio- $(89.3 \% / 80.0 \% / 71.4 \% / 83.3 \% / 32.5)$ the gold standard, TNM staging $(64.3 \% / 50.0 \% / 28.6 \% / 44.0 \% / 3.3)$, as summarized in Table 2. It consists of an LSVM that uses image features, a DT that uses image and clinical features, an LR that uses image and spatial features, and an RF that uses all features.

The results of our study suggest that the characterization of a broad immune cell population, as well as their spatial organization in relation to cancer cells, enables a better estimation of survival compared to the TNM staging system in MIBC patients which, in turn, provides further biological insights. Most of our findings based on whole-slide immunofluorescence images are novel for MIBC, and also corroborate the existing literature on other types of cancer $[19,28,31,61,70,71]$. In particular, we found that the high content of TBs in the invasive frontin, frontout, and tumour core as well as the low number of $\mathrm{CD} 68^{+}$cells and high PD-L1 expression in the invasive frontout, are indicators of bad prognosis $[28,30,61,70]$. The high density of $\mathrm{CD}^{+}, \mathrm{CD}^{+}$, and $\mathrm{CD}^{+} 8^{+}$cells in the invasive frontin, frontout, and tumour core was associated with good prognosis by our models [19,71]. In addition, the high number of $\mathrm{CD}^{+}$and $\mathrm{CD} 68^{+}$cells, as well as the high number of $\mathrm{CD}^{+}$cells clustered within a distance of $20 \mu \mathrm{m}$ from TBs, were linked to good prognosis [31]. Finally, we found that the high density of $\mathrm{CD}_{163}{ }^{+}$cells without PD-L1 expression in frontout is associated with bad prognosis by the DT submodel, whereas the LSVM submodel employed a high number of $\mathrm{CD} 163^{+} \mathrm{PD}_{-\mathrm{L}} 1^{+}$cells in frontout as an indication of good prognosis.

\section{Conclusions}

In summary, we have demonstrated that ML classifiers using image and spatial features from WSIs, combined with clinical features from medical records, can separate MIBC patients into low- and high-risk groups for 5-year prognosis. The present approach outperforms the current clinical staging system, TNM, reinforcing the importance of the standardized quantification of immunological features across WSIs, as well as the adoption of $\mathrm{ML}$ in the clinic. Moreover, our findings show that investigating features from the tumour-immune microenviroment in relation to survival can provide further insights into histopathological studies, thereby contributing to better ways of predicting survivability and enabling a better quality of care.

Supplementary Materials: The following are available online at https:/ / www.mdpi.com/2072-6 694/13/7/1624/s1, Figure S1: Tyramide signal amplification spectra for antibody visualisation, Figure S2: Nuclei detection and epithelium segmentation, Figures S3-S4: Kaplan-Meier curves for MIBC prognosis, Figure S5: Diagram of the DT model, Figure S6: Intersecting features between 
the submodels of the ensemble model, Tables S1-S3: Immunofluorescence primary antibodies and visualization reagents, Table S4: The search space of hyperparameter tuning, Table S5: Nested crossvalidation results on the training data set, Tables S6-S8: Pairwise comparison between TNM staging groups, Table S9-S10: Most important features for MIBC prognosis per classifier. Table S11: The complete list of all image, spatial and clinical features, Table S12: The coefficients of logistic regression for all image and spatial features, Table S13: The mean decrease in Gini index for each feature based on the random forest, Table S14: The feature ranking coefficients of linear support vector machine for all image features.

Author Contributions: C.G.G., P.D.C. and G.S. conceived the idea of this project. D.J.H. and P.D.C. selected the patient samples. C.G.G. and I.P.N. carried out the wet-lab experiments. C.G.G., N.D., N.B., P.D.C. designed different parts of the methodology. C.G.G., N.D. and N.B. implemented the methodology and conducted the corresponding experiments. The manuscript was written by C.G.G. and N.D., and revised by all authors. D.J.H. provided pathology expertise. O.A., G.S., and P.D.C. supervised this project. All authors have read and agreed to the published version of the manuscript.

Funding: This research received financial support from Definiens $\mathrm{GmbH}$ and the Industrial Centre for AI Research in digital Diagnostics (iCAIRD) which is funded by Innovate UK on behalf of UK Research and Innovation (UKRI) [project number: 104690].

Institutional Review Board Statement: The study was conducted according to the guidelines of the Declaration of Helsinki. The corresponding unstained tissue sections were collected from the NHS Lothian NRS BioResource Research Tissue Bank, conforming to protocols approved under the ethical status granted by the East of Scotland Research Ethics Service (Ethical Approval Ref: 10/S1402/33) and with written informed consent from all the patients.

Informed Consent Statement: Informed consent was obtained from all subjects involved in the study.

Data Availability Statement: The data and the code of this study are available from the corresponding authors upon request.

Acknowledgments: We would like to thank Frances Rae, Marie O'Donnell, and the NHS Lothian Tissue Governance Unit for providing the patient clinical data and the tissue samples.

Conflicts of Interest: At the time this study was conducted, N.B. was a full-time employee of Definiens $\mathrm{GmbH}$, a subsidiary of AstraZeneca. G.S. is a full-time employee of Definiens GmbH and owns shares in AstraZeneca. D.J.H. is a member of the Scientific Advisory Board of Definiens.

\section{References}

1. Sanli, O.; Dobruch, J.; Knowles, M.A.; Burger, M.; Alemozaffar, M.; Nielsen, M.E.; Lotan, Y. Bladder cancer. Nat. Rev. Dis. Primers 2017, 3, 17022. [CrossRef]

2. Kamat, A.M.; Hahn, N.M.; Efstathiou, J.A.; Lerner, S.P.; Malmström, P.U.; Choi, W.; Guo, C.C.; Lotan, Y.; Kassouf, W. Bladder cancer. Lancet 2016, 388, 2796-2810. [CrossRef]

3. Knowles, M.A.; Hurst, C.D. Molecular biology of bladder cancer: New insights into pathogenesis and clinical diversity. Nat. Rev. Cancer 2015, 15, 25-41. [CrossRef] [PubMed]

4. Frantzi, M.; Van Kessel, K.E.; Zwarthoff, E.C.; Marquez, M.; Rava, M.; Malats, N.; Merseburger, A.S.; Katafigiotis, I.; Stravodimos, K.; Mullen, W.; et al. Development and validation of urine-based peptide biomarker panels for detecting bladder cancer in a multi-center study. Clin. Cancer Res. 2016, 22, 4077-4086. [CrossRef] [PubMed]

5. Prasad, S.M.; DeCastro, G.J.; Steinberg, G.D. Urothelial carcinoma of the bladder: Definition, treatment and future efforts. Nat. Rev. Urol. 2011, 8, 631. [CrossRef]

6. Robertson, A.G.; Kim, J.; Al-Ahmadie, H.; Bellmunt, J.; Guo, G.; Cherniack, A.D.; Hinoue, T.; Laird, P.W.; Hoadley, K.A.; Akbani, R.; et al. Comprehensive molecular characterization of muscle-invasive bladder cancer. Cell 2017, 171, 540-556. [CrossRef] [PubMed]

7. Glaser, A.P.; Fantini, D.; Shilatifard, A.; Schaeffer, E.M.; Meeks, J.J. The evolving genomic landscape of urothelial carcinoma. Nat. Rev. Urol. 2017, 14, 215. [CrossRef]

8. American Joint Committee on Cancer. AJCC—Cancer Staging Manual. Available online: https://cancerstaging.org/referencestools/deskreferences/Pages/default.aspx (accessed on 10 October 2020).

9. Alifrangis, C.; McGovern, U.; Freeman, A.; Powles, T.; Linch, M. Molecular and histopathology directed therapy for advanced bladder cancer. Nat. Rev. Urol. 2019, 16, 465-483. [CrossRef]

10. Galon, J.; Mlecnik, B.; Bindea, G.; Angell, H.K.; Berger, A.; Lagorce, C.; Lugli, A.; Zlobec, I.; Hartmann, A.; Bifulco, C.; et al. Towards the introduction of the 'Immunoscore'in the classification of malignant tumours. J. Pathol. 2014, 232, 199-209. [CrossRef] 
11. Pagès, F.; Mlecnik, B.; Marliot, F.; Bindea, G.; Ou, F.S.; Bifulco, C.; Lugli, A.; Zlobec, I.; Rau, T.T.; Berger, M.D.; et al. International validation of the consensus Immunoscore for the classification of colon cancer: A prognostic and accuracy study. Lancet 2018, 391, 2128-2139. [CrossRef]

12. Junttila, M.R.; de Sauvage, F.J. Influence of tumour micro-environment heterogeneity on therapeutic response. Nature 2013, 501, 346. [CrossRef]

13. Maman, S.; Witz, I.P. A history of exploring cancer in context. Nat. Rev. Cancer 2018, 18, 359. [CrossRef] [PubMed]

14. Kim, S.H.; Go, S.I.; Song, D.H.; Park, S.W.; Kim, H.R.; Jang, I.; Kim, J.D.; Lee, J.S.; Lee, G.W. Prognostic impact of CD8 and programmed death-ligand 1 expression in patients with resectable non-small cell lung cancer. Br. J. Cancer 2019, $120,547$. [CrossRef]

15. Foerster, F.; Hess, M.; Gerhold-Ay, A.; Marquardt, J.U.; Becker, D.; Galle, P.R.; Schuppan, D.; Binder, H.; Bockamp, E. The immune contexture of hepatocellular carcinoma predicts clinical outcome. Sci. Rep. 2018, 8, 5351. [CrossRef] [PubMed]

16. Wellenstein, M.D.; de Visser, K.E. Cancer-cell-intrinsic mechanisms shaping the tumor immune landscape. Immunity 2018, 48, 399-416. [CrossRef] [PubMed]

17. Fridman, W.H.; Zitvogel, L.; Sautès-Fridman, C.; Kroemer, G. The immune contexture in cancer prognosis and treatment. Nat. Rev. Clin. Oncol. 2017, 14, 717. [CrossRef]

18. Ino, Y.; Yamazaki-Itoh, R.; Shimada, K.; Iwasaki, M.; Kosuge, T.; Kanai, Y.; Hiraoka, N. Immune cell infiltration as an indicator of the immune microenvironment of pancreatic cancer. Br. J. Cancer 2013, 108, 914. [CrossRef]

19. Barnes, T.A.; Amir, E. HYPE or HOPE: The prognostic value of infiltrating immune cells in cancer. Br. J. Cancer 2017, $117,451$. [CrossRef]

20. Lohneis, P.; Sinn, M.; Klein, F.; Bischoff, S.; Striefler, J.K.; Wislocka, L.; Sinn, B.V.; Pelzer, U.; Oettle, H.; Riess, H.; et al. Tumour buds determine prognosis in resected pancreatic ductal adenocarcinoma. Br. J. Cancer 2018, 118, 1485. [CrossRef]

21. Li, L.; Sun, R.; Miao, Y.; Tran, T.; Adams, L.; Roscoe, N.; Xu, B.; Manyam, G.C.; Tan, X.; Zhang, H.; et al. PD-1/PD-L1 expression and interaction by automated quantitative immunofluorescent analysis show adverse prognostic impact in patients with diffuse large B-cell lymphoma having T-cell infiltration: A study from the International DLBCL Consortium Program. Mod. Pathol. 2019, 32, 741-754. [CrossRef]

22. Fumet, J.D.; Richard, C.; Ledys, F.; Klopfenstein, Q.; Joubert, P.; Routy, B.; Truntzer, C.; Gagné, A.; Hamel, M.A.; Guimaraes, C.F.; et al. Prognostic and predictive role of CD8 and PD-L1 determination in lung tumor tissue of patients under anti-PD-1 therapy. Br. J. Cancer 2018, 119, 950. [CrossRef] [PubMed]

23. Goode, E.L.; Block, M.S.; Kalli, K.R.; Vierkant, R.A.; Chen, W.; Fogarty, Z.C.; Gentry-Maharaj, A.; Tołoczko, A.; Hein, A.; Bouligny, A.L.; et al. Dose-response association of CD8+ tumor-infiltrating lymphocytes and survival time in high-grade serous ovarian cancer. JAMA Oncol. 2017, 3, e173290. [PubMed]

24. Cassetta, L.; Pollard, J.W. Targeting macrophages: Therapeutic approaches in cancer. Nat. Rev. Drug Discov. 2018, 11, 889-896. [CrossRef] [PubMed]

25. Biswas, S.K.; Mantovani, A. Macrophage plasticity and interaction with lymphocyte subsets: Cancer as a paradigm. Nat. Immunol. 2010, 11, 889. [CrossRef] [PubMed]

26. Zlobec, I.; Lugli, A. Tumour budding in colorectal cancer: Molecular rationale for clinical translation. Nat. Rev. Cancer 2018, 18, 203-204. [CrossRef]

27. Ueno, H.; Murphy, J.; Jass, J.; Mochizuki, H.; Talbot, I. Tumour budding as an index to estimate the potential of aggressiveness in rectal cancer. Histopathology 2002, 40, 127-132. [CrossRef]

28. Gujam, F.; McMillan, D.; Mohammed, Z.; Edwards, J.; Going, J. The relationship between tumour budding, the tumour microenvironment and survival in patients with invasive ductal breast cancer. Br. J. Cancer 2015, 113, 1066. [CrossRef]

29. Lugli, A.; Kirsch, R.; Ajioka, Y.; Bosman, F.; Cathomas, G.; Dawson, H.; El Zimaity, H.; Fléjou, J.F.; Hansen, T.P.; Hartmann, A.; et al. Recommendations for reporting tumor budding in colorectal cancer based on the International Tumor Budding Consensus Conference (ITBCC) 2016. Mod. Pathol. 2017, 30, 1299. [CrossRef]

30. Brieu, N.; Gavriel, C.G.; Nearchou, I.P.; Harrison, D.J.; Schmidt, G.; Caie, P.D. Automated tumour budding quantification by machine learning augments TNM staging in muscle-invasive bladder cancer prognosis. Sci. Rep. 2019, 9, 5174. [CrossRef] [PubMed]

31. Nearchou, I.P.; Lillard, K.; Gavriel, C.G.; Ueno, H.; Harrison, D.J.; Caie, P.D. Automated analysis of lymphocytic infiltration, tumor budding, and their spatial relationship improves prognostic accuracy in colorectal cancer. Cancer Immunol. Res. 2019, 7, 609-620. [CrossRef]

32. Pardoll, D.M. The blockade of immune checkpoints in cancer immunotherapy. Nat. Rev. Cancer 2012, 12, 252. [CrossRef]

33. Sun, C.; Mezzadra, R.; Schumacher, T.N. Regulation and function of the PD-L1 checkpoint. Immunity 2018, 48, 434-452. [CrossRef]

34. Yu, K.; Zhang, C.; Berry, G.J.; Altman, R.B.; Ré, C.; Rubin, D.L.; Snyder, M. Predicting non-small cell lung cancer prognosis by fully automated microscopic pathology image features. Nat. Commun. 2016, 7, 1-10. [CrossRef]

35. Madabhushi, A.; Lee, G. Image analysis and machine learning in digital pathology: Challenges and opportunities. Med. Image Anal. 2016, 33, 170-175. [CrossRef]

36. Dimitriou, N.; Arandjelović, O.; Harrison, D.J.; Caie, P.D. A principled machine learning framework improves accuracy of stage II colorectal cancer prognosis. NPJ Digit. Med. 2018, 1, 1-9. [CrossRef] 
37. Mokarram, R.; Emadi, M. Classification in Non-linear Survival Models Using Cox Regression and Decision Tree. Ann. Data Sci. 2017, 4, 329-340. [CrossRef]

38. Wang, P.; Li, Y.; Reddy, C.K. Machine Learning for Survival Analysis: A Survey. ACM Comput. Surv. 2017, 51, 1-36. [CrossRef]

39. Brieu, N.; Schmidt, G. Learning size adaptive local maxima selection for robust nuclei detection in histopathology images. In Proceedings of the 2017 IEEE 14th International Symposium on Biomedical Imaging (ISBI 2017), Melbourne, Australia, 18-21 April 2017; pp. 937-941.

40. Criminisi, A.; Shotton, J.; Bucciarelli, S. Decision forests with long-range spatial context for organ localization in CT volumes. In Proceedings of the Medical Image Computing and Computer-Assisted Intervention (MICCAI), London, UK, 20-24 September 2009; pp. 69-80.

41. Brieu, N.; Pauly, O.; Zimmermann, J.; Binnig, G.; Schmidt, G. Slide-specific models for segmentation of differently stained digital histopathology whole slide images. In Proceedings of the Medical Imaging 2016: Image Processing, San Diego, CA, USA, 1-3 March 2016; Volume 9784, p. 978410.

42. Brieu, N.; Gavriel, C.G.; Harrison, D.J.; Caie, P.D.; Schmidt, G. Context-based interpolation of coarse deep learning prediction maps for the segmentation of fine structures in immunofluorescence images. In Proceedings of the Medical Imaging 2018: Digital Pathology, Pathology, Houston, Texas, USA, 6 March 2018; International Society for Optics and Photonics: Bellingham, WA, USA, 2018; Volume 10581, p. 105810.

43. Ronneberger, O.; Fischer, P.; Brox, T. U-net: Convolutional networks for biomedical image segmentation. In Proceedings of the International Conference on Medical Image Computing and Computer-Assisted Intervention, Munich, Germany, 5-9 October 2015; pp. 234-241.

44. Ripley, B.D. Modelling spatial patterns. J. R. Stat. Soc. Ser. B (Methodol.) 1977, 39, 172-192. [CrossRef]

45. Besag, J. Contribution to the discussion on Dr Ripley's paper. JR Stat. Soc. 1977, 39, 193-195.

46. Carstens, J.L.; De Sampaio, P.C.; Yang, D.; Barua, S.; Wang, H.; Rao, A.; Allison, J.P.; LeBleu, V.S.; Kalluri, R. Spatial computation of intratumoral T cells correlates with survival of patients with pancreatic cancer. Nat. Commun. 2017, 8, 15095. [CrossRef]

47. Leung, K.; Elashoff, M.R.; Afifi, A.A. Censoring Issues In Survival Analysis. Annu. Rev. Public Health 1997, 18, 83-104. [CrossRef] [PubMed]

48. Kemi, N.; Eskuri, M.; Kauppila, J.H. Tumour-stroma ratio and 5-year mortality in gastric adenocarcinoma: A systematic review and meta-analysis. Sci. Rep. 2019, 9, 1-6. [CrossRef]

49. Noon, A.; Albertsen, P.; Thomas, F.; Rosario, D.; Catto, J. Competing mortality in patients diagnosed with bladder cancer: evidence of undertreatment in the elderly and female patients. Br. J. Cancer 2013, 108, 1534-1540. [CrossRef]

50. Bergstra, J.; Yamins, D.; Cox, D.D. Making a Science of Model Search: Hyperparameter Optimization in Hundreds of Dimensions for Vision Architectures. In Proceedings of the 30th International Conference on International Conference on Machine Learning, Atlanta, GA, USA, 16-21 June 2013; Volume 28, pp. I-115-I-123.

51. Bergstra, J.; Bengio, Y. Random Search for Hyper-parameter Optimization. J. Mach. Learn. Res. 2012, 13, $281-305$.

52. Wolpert, D.H.; Macready, W.G. No Free Lunch Theorems for Optimization. Trans. Evol. Comp 1997, 1, 67-82. [CrossRef]

53. Harder, N.; Athelogou, M.; Hessel, H.; Brieu, N.; Yigitsoy, M.; Zimmermann, J.; Baatz, M.; Buchner, A.; Stief, C.G.; Kirchner, T.; et al. Tissue Phenomics for prognostic biomarker discovery in low-and intermediate-risk prostate cancer. Sci. Rep. 2018, 8, 4470. [CrossRef]

54. Binnig, G.; Huss, R.; Schmidt, G. Tissue Phenomics: Profiling Cancer Patients for Treatment Decisions; CRC Press: Boca Raton, FL, USA, 2018.

55. Athelogou, M.; Schmidt, G.; Schäpe, A.; Baatz, M.; Binnig, G. Cognition network technology-a novel multimodal image analysis technique for automatic identification and quantification of biological image contents. In Imaging Cellular and Molecular Biological Functions; Springer: Berlin/Heidelberg, Germany, 2007; pp. 407-422.

56. Raschka, S. Model Evaluation, Model Selection, and Algorithm Selection in Machine Learning. arXiv 2018, arXiv:1811.12808.

57. Louppe, G.; Wehenkel, L.; Sutera, A.; Geurts, P. Understanding variable importances in forests of randomized trees. In Advances in Neural Information Processing Systems 26; Burges, C.J.C., Bottou, L., Welling, M., Ghahramani, Z., Weinberger, K.Q., Eds.; Curran Associates, Inc.: Red Hook, NY, USA, 2013; pp. 431-439.

58. Guyon, I.; Weston, J.; Barnhill, S.; Vapnik, V. Gene Selection for Cancer Classification using Support Vector Machines. Mach. Learn. 2002, 46, 389-422. [CrossRef]

59. Gooden, M.J.; de Bock, G.H.; Leffers, N.; Daemen, T.; Nijman, H.W. The prognostic influence of tumour-infiltrating lymphocytes in cancer: A systematic review with meta-analysis. Br. J. Cancer 2011, 105, 93. [CrossRef]

60. Yagi, T.; Baba, Y.; Okadome, K.; Kiyozumi, Y.; Hiyoshi, Y.; Ishimoto, T.; Iwatsuki, M.; Miyamoto, Y.; Yoshida, N.; Watanabe, M.; et al. Tumour-associated macrophages are associated with poor prognosis and programmed death ligand 1 expression in oesophageal cancer. Eur. J. Cancer 2019, 111, 38-49. [CrossRef]

61. Keller, M.D.; Neppl, C.; Irmak, Y.; Hall, S.R.; Schmid, R.A.; Langer, R.; Berezowska, S. Adverse prognostic value of PD-L1 expression in primary resected pulmonary squamous cell carcinomas and paired mediastinal lymph node metastases. Mod. Pathol. 2018, 31, 101. [CrossRef]

62. Masugi, Y.; Abe, T.; Ueno, A.; Fujii-Nishimura, Y.; Ojima, H.; Endo, Y.; Fujita, Y.; Kitago, M.; Shinoda, M.; Kitagawa, Y.; et al. Characterization of spatial distribution of tumor-infiltrating CD8+ T cells refines their prognostic utility for pancreatic cancer survival. Mod. Pathol. 2019, 32, 1495-1507. [CrossRef] 
63. Xue, S.; Song, G.; Yu, J. The prognostic significance of PD-L1 expression in patients with glioma: A meta-analysis. Sci. Rep. 2017, 7, 4231. [CrossRef]

64. Caie, P.; Dimitriou, N.; Nearchou, I.; Arandjelovic, O.; Harrison, D. Artificial Intelligence Driving Automated Pathology: ICAIRD and Beyond. Virchows Arch. 2019, 475, S60.

65. Parra, E.R.; Francisco-Cruz, A.; Wistuba, I.I. State-of-the-art of profiling immune contexture in the era of multiplexed staining and digital analysis to study paraffin tumor tissues. Cancers 2019, 11, 247. [CrossRef]

66. Kather, J.N.; Suarez-Carmona, M.; Charoentong, P.; Weis, C.A.; Hirsch, D.; Bankhead, P.; Horning, M.; Ferber, D.; Kel, I.; Herpel, E.; et al. Topography of cancer-associated immune cells in human solid tumors. Elife 2018, 7, e36967. [CrossRef]

67. König, L.; Mairinger, F.D.; Hoffmann, O.; Bittner, A.K.; Schmid, K.W.; Kimmig, R.; Kasimir-Bauer, S.; Bankfalvi, A. Dissimilar patterns of tumor-infiltrating immune cells at the invasive tumor front and tumor center are associated with response to neoadjuvant chemotherapy in primary breast cancer. BMC Cancer 2019, 19, 120. [CrossRef]

68. Galon, J.; Costes, A.; Sanchez-Cabo, F.; Kirilovsky, A.; Mlecnik, B.; Lagorce-Pagès, C.; Tosolini, M.; Camus, M.; Berger, A.; Wind, P.; et al. Type, density, and location of immune cells within human colorectal tumors predict clinical outcome. Science 2006, 313, 1960-1964. [CrossRef]

69. Galon, J.; Pagès, F.; Marincola, F.M.; Angell, H.K.; Thurin, M.; Lugli, A.; Zlobec, I.; Berger, A.; Bifulco, C.; Botti, G.; et al. Cancer classification using the Immunoscore: A worldwide task force. J. Transl. Med. 2012, 10, 205. [CrossRef]

70. Riihijärvi, S.; Fiskvik, I.; Taskinen, M.; Vajavaara, H.; Tikkala, M.; Yri, O.; Karjalainen-Lindsberg, M.L.; Delabie, J.; Smeland, E.; Holte, H.; et al. Prognostic influence of macrophages in patients with diffuse large B-cell lymphoma: A correlative study from a Nordic phase II trial. Haematologica 2015, 100, 238-245. [CrossRef]

71. Chaput, N.; Svrcek, M.; Aupérin, A.; Locher, C.; Drusch, F.; Malka, D.; Taïeb, J.; Goéré, D.; Ducreux, M.; Boige, V. Tumourinfiltrating CD68+ and CD57+ cells predict patient outcome in stage II-III colorectal cancer. Br. J. Cancer 2013, 109, 1013-1022. [CrossRef] [PubMed] 